\title{
Self-Control Study on the Impact of Buzhong Yiqi Prescription on the Gut Microbiota of Obese Patients with PCOS and Phlegm-Dampness Syndrome Caused by Spleen Deficiency
}

\section{Zhexin Ni}

Changhai Hospital

\section{Wen Cheng}

Changhai Hospital

Jie Ding

Changhai Hospital

Ruipin Yao

Changhai Hospital

\section{Danying Zhang}

Changhai Hospital

Dongxia Zhai

Changhai Hospital

\section{Ling Zhou}

Changhai Hospital

Chaoqin Yu ( $\nabla$ chqyu81@163.com )

Changhai Hospital https://orcid.org/0000-0003-1614-2655

\section{Research}

Keywords: polycystic ovarian syndrome, obesity, traditional Chinese medicine, gut microbiota, metabolomics

Posted Date: December 7th, 2020

DOl: https://doi.org/10.21203/rs.3.rs-117891/v1

License: (9) (1) This work is licensed under a Creative Commons Attribution 4.0 International License. Read Full License 


\section{Abstract}

Background: Gut microbiota disorders have been closely related to polycystic ovarian syndrome (PCOS). Buzhong Yiqi prescription (BZYQ) has a great clinical effect on the treatment of obese patients with PCOS and phlegm-dampness syndrome caused by spleen deficiency (SPSD). This study was performed to explore the alterations in the gut microbiota and fecal metabolites in obese patients with PCOS and SPSD who received BZYQ treatment.

Methods: A total of 50 obese patients with PCOS and SPSD were recruited from the Changhai Hospital in Shanghai and accepted three months of BZYQ treatment. Sex hormone were detected and oral glucose tolerance was tested in the outpatient laboratory before and after the BZYQ treatment. Fecal samples were detected by $16 \mathrm{~S}$ rRNA high-throughput sequencing and nontargeted metabolomic methods to determine the structure of the gut microbiota and metabolites, respectively.

Results: BZYQ could significantly alleviate the serum DHEAS $(p<0.001)$ and T level $(p<0.001)$ in obese patients with PCOS and SPSD. The structure of the gut microbiota changed significantly after the BZYQ treatment. In particular, at the phylum level, the abundance of Spirochaetae was significantly higher after treatment than that before treatment. At the genus level, the abundances of [Eubacterium]_rectale_group, Escherichia-Shigella, and Fusicatenibacter were significantly higher after treatment than those before treatment, but the abundance of Megamonas was significantly lower. A total of 106 differential metabolites and 14 KEGG enrichment pathways were quantified. The disorder in the gut microbiota and fecal metabolites of obese patients with PCOS and SPSD were closely related to hyperandrogenemia and insulin resistance. The level of tetracosanoic acid was negatively correlated with serum DHEA level $(p<0.05)$, while the palmitoleic acid level was negatively correlated with serum $T$ level $(p<0.05)$.

Conclusions: BZYQ could ameliorate the serum androgen level and had an impact on the gut microbiota and metabolites in obese patients with PCOS and SPSD.

Trial registration: Chinese Clinical Trial Registry, ChiCTR-IPR-16009166. Registered 26 September 2016, http://www.chictr.org.cn/showproj.aspx?proj=14956

\section{Background}

Polycystic ovarian syndrome (PCOS) is a chronic disease characterized by reproductive endocrine and metabolic dysfunction. Patients with PCOS often manifest different degrees of menstrual abnormalities, infertility, amount of hair, acne, and obesity. The incidence rate of PCOS can reach 5-15\% [1], and this dysfunction occurs in puberty and childbearing age. The cause of this disease remains unclear [2]. Epidemiological data show that approximately 53.5-85.5\% of patients with PCOS are overweight or obese, with central obesity as the most typical case [3]. Obesity is recognized as the most dangerous factor for insulin resistance. For obese patients with PCOS, weight loss is still one of the important treatments [4]. 
In recent years, research on traditional Chinese medicine (TCM) and intestinal microecology has become a hot topic. Some single-herb or TCM compounds can help maintain the balance of intestinal microecology [4]. Oral herbal decoction and other dosage forms are the most important means of using TCM for the clinical treatment of diseases. This natural therapy plays a role in local parts or even the whole body through the digestive tract, which is the most important region for organism to parasitize microorganisms. Previous studies have shown that the gut microbiota is closely related to PCOS [6-8]. Disordered gut microbiota could increase intestinal permeability by affecting intestinal metabolites, such as lipopolysaccharide, entering the systemic circulation, and causing antigen-antibody reaction in the body. Abnormal intestinal environment could activate the immune system and chronic inflammation and increase serum insulin level and androgen levels in the ovary, interfering with normal follicular development $[9,10]$.

Buzhong Yiqi prescription (BZYQ) was used to treat infertile obese women with phlegm-dampness syndrome caused by spleen deficiency (SPSD) as recorded in the Fu Qing Zhu Nv Ke, an ancient book of TCM in the Qing Dynasty. Previous studies have shown that BZYQ can improve symptoms of diarrhea and indirectly restore host homeostasis by recovering gut microbiota, such as Lactobacillus, Bifidobacterium, Enterococcus, and Bacillus subtilis [11, 12]. Although BZYQ has long been applied to treat obese patients with PCOS and SPSD, the detailed underlying mechanism is still unclear.

Metabolomics is always used conveniently to study metabolism at the molecular level[13]. Fecal metabolites are the cometabolism products of the gut microbiota and host, and these molecules could reflect not only the status of the gut microbiota but also the relationship between commensal bacteria and the host. Metabolomics of PCOS is mainly focused on the metabolism of carbohydrate, lipid, amino acid, and hormones [14]. This thrust indicates that metabolic abnormality is not limited to the ovary but is a systemic metabolic disorder in PCOS, which increases the long-term risk of multiple diseases in patients [15]. In addition, the metabolic disorder of patients with PCOS is significantly affected by their phenotypes [16]. Although this disorder is greatly disturbing, few reports are available on the combined study of gut microbiota and fecal metabolomics of obese patients with PCOS and SPSD, especially those treated with BZYQ.

In this study, $16 \mathrm{~s}$ rRNA sequencing and nontargeted metabolomics were used to analyze the fecal samples of obese patients with PCOS and SPSD and reveal the impact of BZYQ on the gut microbiota and intestinal metabolites.

\section{Methods}

\section{Subjects}

In this study, 50 obese patients with PCOS and SPSD (BMI $\geq 28 \mathrm{~kg} / \mathrm{m}^{2}$ and $16-35$ years old) were recruited from the Traditional Chinese Medicine Gynecology Clinic of Changhai Hospital in Shanghai from June 2016 to November 2017. During the study period, a total of 35 cases did not complete the 
study, and 15 cases completed the study by providing detailed clinical data and gut microbiota sequencing data. To explore the intestinal metabolites, six patients with the best treatment effect were further selected to complete the analysis of the fecal nontargeted metabolomics (Fig. 1). The obesity criteria were based on the consensus on the prevention of Chinese adult obesity [2]. The diagnostic criteria of PCOS were based on those revised in the 2003 Rotterdam Conference. SPSD was assessed according to the criteria set by Li et al. [17]. Patients with the following characteristics were excluded: had used oral contraceptives, antiandrogens, insulin sensitizers in the past 3 months prior to the experiment; pregnant; with other known hyperandrogenemia and ovulation disorders, such as 21 hydroxylase deficiency, congenital adrenal hyperplasia, Cushing's syndrome, androgen secreting tumors, thyroid diseases, and hyperprolactinemia; with mental diseases or organic diseases; had used corticosteroids or sex steroids; with a history of drug and alcohol abuse in the past 2 years prior to the experiment; and had used antibiotics, probiotics, or prebiotics in the past 3 months prior to the experiment. This study was approved by the Chinese Ethics Committee of Registering Clinical Trials (No. ChiCTRCTEC2016050), and each subject voluntarily signed the informed consent form before the trial.

\section{Preparation And Application Of Bzyq}

BZYQ mainly consisted of $30 \mathrm{~g}$ of Huangqi (Hedysarum multijugum Maxim), $15 \mathrm{~g}$ of Fuling [Poria Cocos (Schw.) Wolf, $15 \mathrm{~g}$ of Dangshen (Codonopsis Radix), $12 \mathrm{~g}$ of Baizhu (Atractylodes macrocephala Koidz), $9 \mathrm{~g}$ of Shengma (Cimicifugae Rhizoma), $6 \mathrm{~g}$ of Chaihu (Radix Bupleuri), $9 \mathrm{~g}$ of Chenpi (Citrus reticulata), $15 \mathrm{~g}$ of Danggui (Angelicae sinensis Radix), $15 \mathrm{~g}$ of Shichangpu (Acoritataninowii Rhizoma), $15 \mathrm{~g}$ of Danshen (Radix Salviae), $18 \mathrm{~g}$ of Yinyanghuo (Epimrdii Herba), and $18 \mathrm{~g}$ of Shudihuang (Rehmanniae Radix Praeparata). The herbal medicine was obtained from Caitongdetang Pharmaceutical Co. Ltd. (Shanghai, China) and decocted at high pressure with $2 \mathrm{~L}$ of cold water for $1 \mathrm{~h}$ to prepare the same volume and concentration of liquid medicine bags. The patients were asked to take a liquid medicine bag at $0.5 \mathrm{~h}$ after meals in the morning and evening for 3 months.

\section{Sample Collection}

The samples were detected before and 3 months after BZYQ treatment. The levels of sex hormones in the peripheral blood, including luteinizing hormone (LH), follicle stimulating hormone (FSH), estradiol (E2), testosterone $(T)$, dehydroepiandrosterone sulfate (DHEAS), and prolactin $(P)$, were detected on the third day of the menstrual cycle in the Clinical Laboratory of Changhai Hospital. Fasting blood glucose (FBG) and fasting insulin (FINS, $0 \mathrm{~h}$ ) were detected in the morning after $8 \mathrm{~h}$ of hunger. The insulin levels were detected at $0.5,1,1.5,2$, and $3 \mathrm{~h}$ after the patients ingested a pack of $75 \mathrm{~g}$ of glucose powder with $250 \mathrm{~mL}$ of warm water. The area under the insulin curve (IAUC) was calculated as IAUC $=1 / 2 \times(0 h+3 \mathrm{~h}$ ) $+0.5 \mathrm{~h}+3 / 4 \times 1 \mathrm{~h}+2 \mathrm{~h}$. Homeostatic model assessment for insulin resistance (HOMA-IR) was calculated as $\mathrm{HOMA}-\mathrm{IR}=\mathrm{FBG} \times \mathrm{FINS} / 22.5$, and insulin sensitivity index $(\mathrm{ISI})$ was calculated as ISI $=1 / \mathrm{FBG} \times \mathrm{FINS}$. HOMA-IR $\geq 1.66$ indicated insulin resistance, and $I S I<0.021$ implied a decrease in the insulin sensitivity. 
Fecal samples were collected 3-5 days after menstruation, and the patients received guidance for carbohydrate-based diet ( $300 \mathrm{~g} /$ day) 3 days before sampling. Sterile plastic spoon and sterile test tube were used to collect $\sim 10 \mathrm{~g}$ of fresh fecal samples from each participant. The samples were transported to the laboratory in an ice box within $2 \mathrm{~h}$ from sampling and stored at $-80^{\circ} \mathrm{C}$. Then, $16 \mathrm{~S}$ rRNA gene sequencing was performed to analyze the structure of the gut microbiota, and nontarget metabolomics was applied to analyze the fecal metabolites.

\section{Detection Of Gut Microbiota}

Fecal samples from 15 patients were collected before and after the BZYQ treatment. Sample DNA extraction, PCR amplification, Illumina MiSeq sequencing, and post-processing of data were performed as described in previous studies [9]. The OTU abundance data were used to analyze the intestinal microbial diversity and changes in the abundance before and after BZYQ treatment, and the community composition of each sample at different classification levels was obtained. Mothur software (v.1.30.1, https://mothur.org/) was used to analyze the alpha diversity before and after BZYQ treatment. R language was used to draw a bar map of the community structure at the phylum and genus levels. Beta diversity was analyzed via principal co-ordinate analysis (PCoA). Wilcox rank sum test was used to analyze the differences in the species between the two groups at the phylum and genus levels. Linear discriminant analysis (LDA) was conducted to estimate the influence of the abundance of species on the differences in the gut microbiota after BZYQ treatment.

\section{Nontarget Metabolomic Analysis}

The nontarget metabolomic experimental steps were based on a validated method as previously described [18]. The data were analyzed on the free online platform of Majorbio Cloud Platform (https://www.majorbio.com). Principal component analysis (PCA) and orthogonal partial least squaresdiscriminant analysis (OPLS-DA) were conducted to distinguish the overall differences in the metabolic profiles and find the different metabolites before and after BZYQ treatment $(n=6)$. The metabolites with variable importance for the projection (VIP) greater than 1 and $p$ values less than 0.05 were considered as differential variables. The expression mode of the metabolites in each sample was displayed in the cluster heat map, and the $p$ and VIP values of the metabolites were displayed in a VIP bar chart. The metabolic pathway annotation was carried out through the KEGG database (https://www.kegg.jp/kegg/pathway.html) to obtain the pathways participated by the differential metabolites. Pathway enrichment was analyzed on Python (scipy.stats), and the most relevant biological pathway was selected using Fisher's precise test.

\section{Statistical analysis}

SPSS software (version 21.0) was used for statistical analysis. Paired t test was used to analyze the quantitative demographic and clinical data with normal distribution, and the data were expressed as 
mean with standard deviation. Wilcoxon rank sum test was used to analyze the quantitative sequencing data with nonnormal distribution, and the $p$ values were checked multiple times using Benjamini and Hochberg false discovery rate. A double-tailed $p<0.05$ indicated statistically significant difference.

\section{Results}

Comparison of clinical data of obese patients with PCOS and SPSD before and after BZYQ treatment

After BZYQ treatment for 3 months, no significant differences were found in the BMI (Fig. 2a), WHR (Fig. 2b), E2 (Fig. 2C), FSH (Fig. 2d), PRL (Fig. 2e), FBG (Fig. 2f), IAUC (Fig. 2g), ISI (Fig. 2h), and insulin levels (Fig. S) compared with those before treatment. The LH (Fig. 2i) and HOMA-IR (Fig. 2j) indices of the patients showed a decreasing trend, but no statistical significance was noted. The DHEAS (Fig. 2k) and T (Fig. 2l) levels decreased significantly.

\section{Changes In The Gut Microbiota Structure}

In this study, 16S rRNA high-throughput sequencing was used to detect the structures of the gut microbiota before and after BZYQ treatment $(n=15)$. The results showed no significant difference in the alpha diversity (Sobs, Chao, Shannon, and Simpson) and beta diversity (PCA and PCoA) before and after BZYQ treatment. At the phylum level, BZYQ treatment increased the abundance of Firmicutes, Bacteroidetes, Proteobacteria, Actinobacteria, and the ratio of Firmicutes/Bacteroidetes (2.0 vs. 1.91) but decreased the abundances of Fusobacteria and Verrucomicrobia. However, no significant differences were found on these phyla. Although the abundance of Spirochaetae increased significantly after BZYQ treatment $(p=0.0006)$, the sample was limited and short of clinical significance (Fig. 3a).

The top 20 species were selected in accordance with abundance for comparison due to the large number of species at the genus level. BZYQ treatment increased the abundances of [Eubacterium]_rectale_group, Escherichia-Shigella, unclassified_f_Lachnospiraceae, and Fusicatenibacter $(p<0.05)$ but decreased the abundance of Megamonas ( $p<0.05$, Fig. 3b). Prior to the BZYQ treatment, 26 unique genera were found in the patients compared with those after BZYQ treatment, and Mitsuokella was the most abundant, accounting for $64.11 \%$ (Fig. 3c). After BZYQ treatment, 13 unique genera were found, of which Edwardsiella was the most abundant at $28.05 \%$ (Fig. 3d). Furthermore, the LDA scores showed that Dialister, Holdemania, Megamonas, Ruminiclostridium_9, and vadinBC27_wastewater_sludge_group were the characteristic species before the BZYQ treatment. After the BZYQ treatment, 19 genera, such as Fusicatenibacter, Blautia, and Dorea, were the characteristic species (Fig. 3e).

\section{Comparison Of Fecal Metabolites Before And After Bzyq Treatment}


PCA (Fig. 4a), OPLS-DA (Fig. 4b), and model validation (Fig. 4c) results showed that the samples before and after BZYQ treatment $(n=6)$ were significantly grouped. In this study, 962 different ion peaks were found, and 106 different metabolites (VIP > 1, $p<0.05$ ) were quantified (Fig. 4d). KEGG pathway analysis demonstrated the involvement of 10 different metabolites, such as taurocholic acid, palmitic acid, and stearic acid (Fig. 5a, Table 1). The 10 differential metabolites participated in 26 KEGG pathways, of which the lipid metabolism pathway contained the most differential metabolites (Fig. 5b). The differential metabolites were significantly enriched in 14 KEGG pathways, including the biosynthesis of unsaturated fatty acids $(p<0.001)$, fatty acid biosynthesis $(p<0.001)$, cutin, suberine, and wax biosynthesis $(p<$ $0.001)$ and biosynthesis of plant secondary metabolites $(p<0.001$, Fig. $5 c)$. 
Table 1

Relative abundance of 10 differential metabolites involved in KEGG pathway

\begin{tabular}{|c|c|c|c|c|c|c|c|}
\hline \multirow[t]{2}{*}{ Metabolite } & \multirow[t]{2}{*}{ Formula } & \multicolumn{2}{|c|}{ Groups (mean with SD) } & \multirow[t]{2}{*}{ VIP } & \multirow[t]{2}{*}{ FC } & \multirow{2}{*}{$p_{\text {value }}^{p}$} & \multirow{2}{*}{$\begin{array}{l}\text { AUC } \\
\text { (Cl) }\end{array}$} \\
\hline & & $\begin{array}{l}\text { before ( } \mathrm{n} \\
=6 \text { ) }\end{array}$ & $\begin{array}{l}\text { after }(n= \\
6)\end{array}$ & & & & \\
\hline Palmitic acid & C16H32O2 & $\begin{array}{l}9490 \pm \\
933\end{array}$ & $\begin{array}{l}12219 \pm \\
1930\end{array}$ & 8.57 & 0.78 & 0.01 & $\begin{array}{l}0.92 \\
(0.74- \\
1)\end{array}$ \\
\hline Taurocholic acid & $\mathrm{C} 26 \mathrm{H} 45 \mathrm{NO} 7 \mathrm{~S}$ & $\begin{array}{l}3408 \pm \\
2030\end{array}$ & $\begin{array}{l}1106 \pm \\
797\end{array}$ & 7.21 & 3.08 & 0.027 & $\begin{array}{l}0.81 \\
(0.53- \\
1)\end{array}$ \\
\hline Stearic acid & $\mathrm{C} 18 \mathrm{H} 3602$ & $\begin{array}{l}8791 \pm \\
585\end{array}$ & $\begin{array}{l}10505 \pm \\
1413\end{array}$ & 6.90 & 0.84 & 0.021 & $\begin{array}{l}0.86 \\
(0.58- \\
1)\end{array}$ \\
\hline $\begin{array}{l}\text { Sphingosine 1- } \\
\text { phosphate }\end{array}$ & C18H38N05P & $960 \pm 235$ & $\begin{array}{l}1707 \pm \\
506\end{array}$ & 4.64 & 0.56 & 0.008 & $\begin{array}{l}0.89 \\
(0.69- \\
1)\end{array}$ \\
\hline Palmitoleic acid & $\mathrm{C} 16 \mathrm{H} 3002$ & $\begin{array}{l}1647 \pm \\
339\end{array}$ & $\begin{array}{l}2299 \pm \\
529\end{array}$ & 3.96 & 0.72 & 0.029 & $\begin{array}{l}0.86 \\
(0.64- \\
1)\end{array}$ \\
\hline Eicosenoic acid & $\mathrm{C} 2 \mathrm{OH} 38 \mathrm{O} 2$ & $583 \pm 208$ & $976 \pm 297$ & 3.21 & 0.60 & 0.024 & $\begin{array}{l}0.86 \\
(0.64- \\
1)\end{array}$ \\
\hline Erucic acid & $\mathrm{C} 22 \mathrm{H} 42 \mathrm{O} 2$ & $93 \pm 93$ & $510 \pm 447$ & 3.10 & 0.18 & 0.049 & $\begin{array}{l}0.86 \\
(0.64- \\
1)\end{array}$ \\
\hline Tetracosanoic acid & $\mathrm{C} 24 \mathrm{H} 48 \mathrm{O} 2$ & $423 \pm 45$ & $534 \pm 55$ & 1.82 & 0.79 & 0.003 & $\begin{array}{l}0.92 \\
(0.74- \\
1)\end{array}$ \\
\hline Behenic acid & $\mathrm{C} 22 \mathrm{H} 44 \mathrm{O} 2$ & $276 \pm 18$ & $346 \pm 36$ & 1.47 & 0.80 & 0.002 & $\begin{array}{l}0.92 \\
(0.74- \\
1)\end{array}$ \\
\hline Xanthine & $\mathrm{C} 5 \mathrm{H} 4 \mathrm{~N} 4 \mathrm{O} 2$ & $81 \pm 49$ & $18 \pm 11$ & 1.20 & 4.46 & 0.011 & $\begin{array}{l}0.97 \\
(0.9-1)\end{array}$ \\
\hline
\end{tabular}

Correlation analysis among the gut microbiota, fecal metabolites, and serum sex hormones after BZYQ treatment

A significant positive correlation was observed between the abundance of Paraprevotella and serum LH level in the patients after BZYQ treatment $(p<0.01)$. The serum DHEAS and T levels were negatively correlated with the abundances of Lachnospiraceae_NC2004_group and Faecalibacterium $(p<0.05)$ but 
positively correlated with the abundance of [Ruminococcus]_gnavus_group $(p<0.05)$. HOMA-IR was positively correlated with the abundance of Blautia $(p<0.01$, Fig. 6a).

The correlation between the 10 fecal metabolites involved in the KEGG pathways and the top 50 genera was analyzed in accordance with their abundance. These fecal metabolites, except for Sphingosine_1_phosphate, had significant correlations with specific genera (Fig. 6b). In particular, the abundance of Bacteroides was significantly correlated with the palmitoleic acid level $(p<0.001)$, and abundance of the [Eubacterium]_ventriosum_group was positively correlated with the tetracosanoic acid level $(p<0.001$, Fig. $6 b)$. The results showed that combined with changes in the fecal metabolites and bacterial abundance after BZYQ treatment, BZYQ had an effect on the abundance of the gut microbiota and fecal metabolite level, which showed significant correlation (Fig. 6c).

In addition, the tetracosanoic acid level was negatively correlated with the serum DHEAS level $(p<0.05)$. Palmitoleic acid level was negatively correlated with the serum T level $(p<0.05)$. The levels of eicosenoic acid, palmitic acid, and erucic acid were all positively correlated with HOMA-IR $(p<0.05$, Fig. $6 \mathrm{~d})$.

\section{Discussion}

PCOS is an endocrine metabolic disorder with multiple causes and polymorphic clinical symptoms. Chinese medicine can obviously improve the clinical symptoms of obese patients with PCOS, with small side effects, without drug dependence, and with other advantages [19]. BZYQ is an effective prescription for the treatment of obese patients with PCOS and SPSD [20], but its mechanism on the intestinal environment has not been reported yet. In this study, the impact of BZYQ on the gut microbiota and fecal metabolites of obese patients with PCOS and SPSD were discussed through the study of intestinal microecology and nontargeted metabolomics.

Gut microbiota is a general term for sojourn microorganisms in the human intestine, which has physiological functions, such as participating in the body's nutritional metabolism, antagonizing pathogenic microorganisms, immunity, and maintaining the balance of the internal environment [21]. In TCM theory, the gut microbiota is closely related to the physiological function of the "spleen" [22]. When the gut microbiota is disturbed, gastrointestinal discomfort, decline in digestive and absorption function, and other clinical manifestations will be manifested, similar to the spleen deficiency syndrome in TCM [23]. Obese patients with PCOS often have spleen deficiency symptoms, including obesity, fatigue, loss of appetite, and thin stool. In recent years, increasing studies have confirmed that spleen-invigorating TCM compounds help regulate the gut microbiota and maintain the balance in intestinal microecology [24]. $B Z Y Q$ is one of the spleen-invigorating TCM prescriptions and have a remarkable impact on recovering the gut microbiota of the host. The regulation of gut microbiota may be one of the mechanisms of the treatment for the spleen deficiency syndrome.

The results of the 16S rRNA high-throughput sequencing demonstrated that the composition structure of the gut microbiota in obese patients with PCOS and SPSD at the phylum and genus levels had changed significantly. At the phylum level, the abundance of Spirochaetae increased significantly after BZYQ 
treatment, but its abundance in the sample was too small, and the clinical significance was small. At the genus level, the bacteria in the top 20 abundances were compared. After BZYQ treatment, the abundances of [Eubacterium]_rectale_group, Escherichia-Shigella, unclassified_f_Lachnospiraceae, and Fusicatenibacter increased significantly, whereas the abundance of Megamonas decreased significantly. [Eubacterium]_rectale_group is a bacterium that produces butyrate (an anti-inflammatory compound) and plays a key role in fighting inflammation [25]. Cattaneo et al. [26] found that the serum levels of proinflammatory cytokines IL-1 $\beta, N L R P 3$, and CXCL2 in the elderly with cognitive impairment cerebral amyloidosis were negatively correlated with the abundance of [Eubacterium]_rectale_group. The increased abundance of [Eubacterium]_rectale_group in patients with inflammatory bowel disease indicates the enhancement of the anti-TNF-a [27].

PCOS is a chronic inflammatory disease, and chronic nonspecific inflammatory factors affect follicle development, resulting in infertility and adverse pregnancy outcomes by influencing ovarian function, androgen synthesis in vivo, and insulin resistance $[28,29]$. The present study suggested that BZYQ may improve chronic inflammation in obese patients with PCOS and SPSD by improving the abundance of [Eubacterium]_rectale_group]. Megamonas could produce short-chain fatty acids (SCFA) [30], which are converted from indigestible carbohydrates by the gut microbiota [31]. Meanwhile, den Besten et al. [32] found that SCFA could activate peroxisome proliferator-activated receptor- $\gamma$ in the liver and muscle, thereby regulating uptake of glucose and oxidation of fatty acid. In addition, the gut microbiota could influence the insulin sensitivity by SCFA-mediating inflammatory responses [33]. In the present study, the abundance of Megamonas in the patients decreased after BZYQ treatment, which was beneficial for reducing intestinal permeability and maintaining intestinal homeostasis. The Lefse multi-level differential analysis of species showed that the characteristic genera were Dialister, Holdemania, Megamonas, Ruminiclostridium_9, and vadinBC27_wastewater_sludge_group 5 species before treatment, but the characteristic genera after treatment were Fusicatenibacter, Blautia, and Dorea. Studies have confirmed that lipopolysaccharides produced by Gram-negative bacteria are key molecules involved in the early development of inflammation and metabolic diseases, and these bacteria have an endotoxin effect. Gram-negative bacteria could stimulate the production of many inflammatory factors and produce chronic systemic inflammation by binding to the CD14-toll receptor 4 complex on the surface of innate immune cells [34]. These bacteria could also promote insulin resistance via the phosphorylation of insulin receptor substrate 1 through signaling pathways, such as nuclear factor kB. Thus, Dialister was speculated to be associated with chronic inflammation and insulin resistance in obese patients with PCOS and SPSD.

BZYQ also exhibited implications on the fecal metabolites of obese patients with PCOS and SPSD. The contents of taurocholic acid and xanthine were upregulated after BZYQ treatment, while eight differential metabolites, such as palmitic acid, stearic acid, and sphingosine, were downregulated according to the nontargeted metabolomic studies. Taurocholic acid is a primary bile acid that binds amide to the amino group of the cholic acid carboxyl group and taurine. Previous studies [35] have proven that taurocholic acid has a significant inhibitory effect on acute and chronic inflammations, and its mechanism of action is related to its inhibition of macrophage infiltration and the production of pro-inflammatory adipokines. 
Palmitic acid is a long-chain saturated fatty acid, which is an important component of blood lipids. Some studies [36] have found that free palmitic acid can induce stress of the endoplasmic reticulum and then induce $\beta$ cell apoptosis and inhibit insulin synthesis and secretion when glucose concentration is too high. Moreover, in the macrophages, palmitic acid induces the inflammatory responses by increasing FABP4/aP2 protein expression [37]. BZYQ may improve the disorder of glucose metabolism and chronic inflammation of obese patients with PCOS and SPSD by reducing palmitic acid abundance. Sphingosine and its metabolic enzymes are key mediators in the human body. Sphingosine kinase and its lipid product, namely, sphingosine 1-phosphate, are involved in signal transduction and diseases, especially in chronic inflammatory diseases and autoimmunity. These molecules play an important role in the occurrence and development of the disease. In the present study, the abundance of sphingosine in obese patients with PCOS and SPSD was reduced after BZYQ treatment, and this event may help reduce the inflammatory response in the body.

In addition, the differential metabolites were significantly enriched in 14 KEGG pathways, such as the biosynthesis of unsaturated fatty acids, fatty acids, cutin, and wax. Most enrichment pathways were lipid metabolism enrichment pathways. The pathogenesis of abnormal lipid metabolism was related to ApoA1, the related regulators of lipid metabolism, adiponectin, leptin, and endogenin [38]. Obirikorang et al. [39] found that a decrease in total blood adiponectin levels can induce IR, obesity, and type 2 diabetes. The gut microbiota is affected by sex hormones and also has an impact on the serum the sex hormone levels [40]. Through the correlation analysis of the gut microbiota-fecal metabolites-serum sex hormones and HOMA-IR values, the abundances of the different bacterial groups after BZYQ treatment were adjusted in different directions, and most of the metabolites involved in KEGG showed an upward trend. Among these bacteria, the abundance of Paraprevotella in obese patients with PCOS and SPSD was positively correlated with serum LH levels, and the correlation was significant. The abundances of Lachnospiraceae_NC2004_group and Faecalibacterium were negatively correlated with the serum DHEAS and T levels, whereas the abundance of [Ruminococcus]_gnavus_group was positively correlated with the aforementioned parameters. The abundance of Blautia was positively correlated with HOMA-IR values. Published studies have proven that Blautia contributes to maintaining glucose stability and its dysregulation impairs the intracellular insulin signaling [41]. The abundance of [Eubacterium]_ventriosum_group was positively correlated with tetracosanoic_acid, but this acid was negatively correlated with serum DHEAS levels. The abundance of Bacteroides was positively correlated with serum T levels but negatively correlated with serum levels. A linear relationship existed among the gut microbiota, fecal metabolite, and hyperandrogenemia. BZYQ may have improved the disorder of hyperandrogen by regulating the abundance of Bacteroides, [Eubacterium]_ventriosum_group, and their differential fecal metabolites.

Although the number of subjects was relatively small, the authors complied strictly by controlling the inclusion and exclusion criteria and excluded the most factors with potential impact on the gut microbiota. In addition, the authors conducted diet guidance and trained sampling method for subjects before sampling. Hence, heterogeneity was greatly reduced in the group, so the authors are confident that the results are greatly meaningful. In this study, fecal metabolomics combined with gut microbiota was 
used to explore the relationship between the intestinal environment and clinical parameters in obese patients with PCOS and SPSD who were treated with BZYQ.

\section{Conclusions}

BZYQ could ameliorate the serum DHEAS and T level and had an impact on the gut microbiota and metabolites in obese patients with PCOS and SPSD. Relationships existed among the gut microbiota, fecal metabolites, and hyperandrogenism. BZYQ could ameliorate a part of the endocrine disorders in these patients, and this process may be achieved by regulating the abundances of Bacteroides, [Eubacterium]_ventriosum_group, and Blautia and the level of important fecal metabolites, such as palmitoleic acid, tetracosanoic acid, and eicosenoic acid.

\section{Abbreviations}

PCOS polycystic ovarian syndrome

BZYQ Buzhong Yiqi prescription

SPSD Syndrome of phlegmdampness due to spleen deficiency

TCM traditional Chinese medicine

BMI body mass index

LH luteinizing hormone

FSH follicle stimulating hormone

E2 estradiol (E2)

T testosterone

DHEAS dehydroepiandrosterone sulfate

P prolactin

FBG fasting blood glucose

FINS fasting insulin

IAUC area under the insulin curve

HOMA-IR homeostatic model assessment for insulin resistance

ISI insulin sensitivity index 
LDA linear discriminant analysis

PCA principal component analysis

OPLS-DA orthogonal partial least squares-discriminant analysis

VIP variable importance for the projection

FDR false discovery rate

SCFA short-chain fatty acids

\section{Declarations}

\section{Ethics approval and consent to participate}

This study was approved by the Chinese Ethics Committee of Registering Clinical Trials, ChiECRCT (No. ChiCTRCTEC2016050). All participants voluntarily signed the informed consent before participating.

\section{Consent for publication}

Consents for publication were obtained from all participants.

\section{Availability of data and materials}

The raw clinical data of patients is not available due to hospital privacy regulations. The sequencing and LC-MS data are availability on the free online platform of Majorbio Cloud Platform (www.majorbio.com) with the account provided by corresponding author on reasonable request.

\section{Competing interests}

The authors declare that they have no competing interests.

\section{Funding}

This work was supported by the National Natural Science Foundation of China (82004408), Shanghai Sailing Program (20YF1448600), Leading Project of Traditional Chinese Medicine of Shanghai Science and Technology Committee (19401930200) and Youth Initiation Fund of Changhai Hospital (CH201710). These Foundations supported the fecal samples collection and detection of 16S rRNA high-throughput sequencing and non-targeted metabolomic methods, but had no role in the design of the study and collection, analysis, and interpretation of data and in writing the manuscript.

\section{Authors' contributions}

CY and LZ conceived and supervised the study. ZN, WC, JD, RY, DZ, and DZ recruited patients and collected samples. ZN and WC analyzed data and drafted the manuscript. All authors approved the 
manuscript at submission format.

\section{Acknowledgements}

We thank all the doctors, nurses, and laboratorian for their assistance, and all the patients for their contribution to this study.

\section{References}

1. Thackray VG. Sex. Microbes, and Polycystic Ovary Syndrome. Trends Endocrinol Metab. 2019;30:54-65. https://pubmed.ncbi.nlm.nih.gov/30503354/.

2. Yu J, Yu CQ, Cao Q, Wang L, Wang WJ, Zhou LR, et al. Consensus on the integrated traditional Chinese and Western medicine criteria of diagnostic classification in polycystic ovary syndrome (draft). J Integr Med. 2017;15:102-9. https://pubmed.ncbi.nlm.nih.gov/28285615/.

3. Lim SS, Davies MJ, Norman RJ, Moran LJ. Overweight, obesity and central obesity in women with polycystic ovary syndrome: a systematic review and meta-analysis. Hum Reprod Update. 2012;18:618-37. https://pubmed.ncbi.nIm.nih.gov/22767467/.

4. Yang J, Chon TY, Bauer BA. Use of Acupuncture in Overweight/Obese Women with Polycystic Ovary Syndrome. Med Acupunct. 2019;31:267-8. https://pubmed.ncbi.nIm.nih.gov/31624524/.

5. Feng W, Ao H, Peng C, Yan D. Gut microbiota, a new frontier to understand traditional Chinese medicines. Pharmacol Res. 2019;142:176-91. https://pubmed.ncbi.nlm.nih.gov/30818043/.

6. Zeng B, Lai Z, Sun L, Zhang ZB, Yang JH, Li ZX, et al. Structural and functional profiles of the gut microbial community in polycystic ovary syndrome with insulin resistance (IR-PCOS): a pilot study. Res Microbiol. 2019;170:43-52. https://pubmed.ncbi.nlm.nih.gov/30292647/.

7. Torres PJ, Siakowska M, Banaszewska B, Pawelczyk L, Duleba AJ, Kelley ST, et al. Gut Microbial Diversity in Women With Polycystic Ovary Syndrome Correlates With Hyperandrogenism. J Clin Endocrinol Metab. 2018;103:1502-11. https://pubmed.ncbi.nlm.nih.gov/29370410/.

8. Kelley ST, Skarra DV, Rivera AJ, Thackray VG. The Gut Microbiome Is Altered in a Letrozole-Induced Mouse Model of Polycystic Ovary Syndrome. PLoS One. 2016;11:e0146509. https://pubmed.ncbi.nlm.nih.gov/26731268/.

9. Zhou L, Ni Z, Cheng W, Yu J, Shun S, Zhai DX, et al. Characteristic gut microbiota and predicted metabolic functions in women with PCOS. Endocr Connect. 2020;9:63-73. https://pubmed.ncbi.nlm.nih.gov/31972546/.

10. Qi X, Yun C, Sun L, Xia JL, Wu Q, Wang Y, et al. Gut microbiota-bile acid-interleukin-22 axis orchestrates polycystic ovary syndrome. Nat Med. 2019;25:1225-33. https://pubmed.ncbi.nlm.nih.gov/31332392/.

11. Zhao XM, Li N, Du CX, Wang Q. Intervention Effect on Modified Buzhong Yiqi Decoction in Treating Rats of Liver Stagnation and Spleen Deficiency Syndrome. Acta Chinese Medicine. 2017;32:1475- 
1478. http://zyx.cbpt.cnki.net/WKB2/WebPublication/paperDigest.aspx?paperID=33438bc7-c81b44be-a99a-d187a65abf33.

12. Feng $X Z$, Zhang $Y N$, Jiang $X$, Wang HG. Research of modified Bu zhongyiqitang on promote the growth of intestinal probiotics. Chin J Microecol. 2008;20:159-160.

http://d.wanfangdata.com.cn/conference/7074013.

13. Halama A, Aye MM, Dargham SR, Kulinski M, Suhre K, Atkin SL. Metabolomics of Dynamic Changes in Insulin Resistance Before and After Exercise in PCOS. Front Endocrinol (Lausanne). 2019;10:116. https://pubmed.ncbi.nlm.nih.gov/30873121/.

14. Chang AY, Lalia AZ, Jenkins GD, Dutta T, Carter RE, Singh RJ, et al. Combining a nontargeted and targeted metabolomics approach to identify metabolic pathways significantly altered in polycystic ovary syndrome. Metabolism. 2017;71:52-63. doi:10.1016/j.metabol.2017.03.002.

15. Meier RK. Polycystic Ovary Syndrome. Nurs Clin North Am. 2018;53:407-20. https://pubmed.ncbi.nlm.nih.gov/30100006/.

16. Bil E, Dilbaz B, Cirik DA, Ozelci R, Ozkaya E, Dilbaz S. Metabolic syndrome and metabolic risk profile according to polycystic ovary syndrome phenotype. J Obstet Gynaecol Res. 2016;42:837-43. https://pubmed.ncbi.nlm.nih.gov/27071345/.

17. Li YQ, Hou LH, Gao JJ, Wang YY. Chinese Medicine Syndrome Distribution and Study on Characteristics of Sexual Hormones and Glucose Metabolism of Polycystic Ovarian Syndrome. Chinese Archives of Traditional Chinese Medicine. 2016;34:579-82. DOI:10.13193/j.issn.16737717.2016.03.020.

18. Liao X, Song L, Zeng B, Liu BY, Qiu YY, Qu H, et al. Alteration of gut microbiota induced by DPP-4i treatment improves glucose homeostasis. EBioMedicine. 2019;44:665-74. https://pubmed.ncbi.nlm.nih.gov/30922964/.

19. Ong M, Peng J, Jin X, Qu X. Chinese Herbal Medicine for the Optimal Management of Polycystic Ovary Syndrome. Am J Chin Med. 2017;45:405-22. https://pubmed.ncbi.nlm.nih.gov/28359195/.

20. Li CG, Zhou L, Li S, Li X. Clinical Observation of Jiawei Buzhong Yiqi Decoction in the Treatment of Obese Polycystic Ovarian Syndrome. Medicinal Plant. 2018;9:94-96. http://mdpt.cbpt.cnki.net/WKB2/WebPublication/paperDigest.aspx?paperID=dc117584-5371-48ce8b75-450cb62de6a8.

21. Wang W, Li Y, Wu Q, Pan X, He X, Ma X. High-throughput sequencing study of the effect of transabdominal hysterectomy on intestinal flora in patients with uterine fibroids. BMC Microbiol. 2020;20:98. https://pubmed.ncbi.nlm.nih.gov/32299359/.

22. Peng Y, Zhang S, Liu Z, Ji J, Wu CF, Yang JY, et al. Gut microbiota and Chinese medicine syndrome: altered fecal microbiotas in spleen (Pi)-deficient patients. J Tradit Chin Med. 2020;40:137-43. https://pubmed.ncbi.nlm.nih.gov/32227775/.

23. Ma S, Jiang Y, Zhang B, Pang J, Xu XY, Sun JZ, et al. Comparison of the Modulatory Effect on Intestinal Microbiota between Raw and Bran-Fried Atractylodis Rhizoma in the Rat Model of Spleen- 
Deficiency Syndrome. Int J Environ Res Public Health. 2019;16:3183.

https://pubmed.ncbi.nlm.nih.gov/31480462/.

24. Yong W, Zhang L, Chen Y, Li J, Liu Y, Zhang Z. Jianpi Huatan Tongfu granule alleviates inflammation and improves intestinal flora in patients with acute exacerbation of chronic obstructive pulmonary disease. J Int Med Res. 2020;48:300060520909235. https://pubmed.ncbi.nlm.nih.gov/32295442/.

25. Pryde SE, Duncan SH, Hold GL, Stewart CS, Flint HJ. The microbiology of butyrate formation in the human colon. FEMS Microbiol Lett. 2002;217:133-9. https://pubmed.ncbi.nlm.nih.gov/12480096/.

26. Cattaneo A, Cattane N, Galluzzi S, Provasi S, Lopizzo N, Festari C, et al. Association of brain amyloidosis with pro-inflammatory gut bacterial taxa and peripheral inflammation markers in cognitively impaired elderly. Neurobiol Aging. 2017;49:60-8.

https://pubmed.ncbi.nlm.nih.gov/27776263/.

27. Chorell E, Andersson E, Evans ML, Jain N, Götheson A, Åden J, et al. Bacterial Chaperones CsgE and CsgC Differentially Modulate Human a-Synuclein Amyloid Formation via Transient Contacts. PLoS One. 2015;10:e0140194. https://pubmed.ncbi.nlm.nih.gov/26465894/.

28. Ma QW, Tan Y. Effectiveness of co-treatment with traditional Chinese medicine and letrozole for polycystic ovary syndrome: a meta-analysis. J Integr Med. 2017;15:95-101. https://pubmed.ncbi.nlm.nih.gov/28285614/.

29. Shaaban Z, Khoradmehr A, Amiri-Yekta A, Jafarzadeh Shirazi MR, Tamadon A. Pathophysiologic mechanisms of obesity- and chronic inflammation-related genes in etiology of polycystic ovary syndrome. Iran J Basic Med Sci. 2019;22:1378-86. https://pubmed.ncbi.nlm.nih.gov/32133054/.

30. Shimizu J, Kubota T, Takada E, Takai K, Fujiwara N, Arimitsu N, et al. Relative abundance of Megamonas hypermegale and Butyrivibrio species decreased in the intestine and its possible association with the $T$ cell aberration by metabolite alteration in patients with Behcet's disease (210 characters). Clin Rheumatol. 2019;38:1437-45. https://pubmed.ncbi.nlm.nih.gov/30628011/.

31. Morrison DJ, Preston T. Formation of short chain fatty acids by the gut microbiota and their impact on human metabolism. Gut Microbes. 2016;7:189-200.

https://pubmed.ncbi.nlm.nih.gov/26963409/.

32. den Besten G, Bleeker A, Gerding A, van Eunen K, Havinga R, van Dijk TH, et al. Short-Chain Fatty Acids Protect Against High-Fat Diet-Induced Obesity via a PPARY-Dependent Switch From Lipogenesis to Fat Oxidation. Diabetes. 2015;64:2398-408. https://pubmed.ncbi.nlm.nih.gov/25695945/.

33. Zhao X, Jiang Y, Xi H, Chen L, Feng X. Exploration of the Relationship Between Gut Microbiota and Polycystic Ovary Syndrome (PCOS): a Review. Geburtshilfe Frauenheilkd. 2020;80:161-71. https://pubmed.ncbi.nlm.nih.gov/32109968/.

34. Saad MJ, Santos A, Prada PO. Linking Gut Microbiota and Inflammation to Obesity and Insulin Resistance. Physiology (Bethesda). 2016;31:283-93. https://pubmed.ncbi.nlm.nih.gov/27252163/.

35. Murakami S. The physiological and pathophysiological roles of taurine in adipose tissue in relation to obesity. Life Sci. 2017;186:80-6. https://pubmed.ncbi.nlm.nih.gov/28801262/. 
36. Yalçin A, Şarkici G, Kolaç UK. PKR inhibitors suppress endoplasmic reticulum stress and subdue glucolipotoxicity-mediated impairment of insulin secretion in pancreatic beta cells. Turk J Biol. 2020;44:93-102. https://pubmed.ncbi.nlm.nih.gov/32256145/.

37. Korbecki J, Bajdak-Rusinek K. The effect of palmitic acid on inflammatory response in macrophages: an overview of molecular mechanisms. Inflamm Res. 2019;68:915-32. https://pubmed.ncbi.nlm.nih.gov/31363792/.

38. Chazenbalk G, Chen YH, Heneidi S, Lee JM, Pall M, Ida Chen YD, et al. Abnormal expression of genes involved in inflammation, lipid metabolism, and Wnt signaling in the adipose tissue of polycystic ovary syndrome. J Clin Endocrinol Metab. 2012;97:E765-70.

https://pubmed.ncbi.nlm.nih.gov/22344199/.

39. Obirikorang C, Owiredu WKBA, Adu-Afram S, Acheampong E, Asamoah EA, Antwi-Boasiakoh EK, et al. Assessing the variability and predictability of adipokines (adiponectin, leptin, resistin and their ratios) in non-obese and obese women with anovulatory polycystic ovary syndrome. BMC Res Notes. 2019;12:513. https://pubmed.ncbi.nIm.nih.gov/31416473/.

40. Insenser M, Murri M, Del Campo R, Martínez-García M, Fernández-Durán E, Escobar-Morreale HF. Gut Microbiota and the Polycystic Ovary Syndrome: Influence of Sex, Sex Hormones, and Obesity. J Clin Endocrinol Metab. 2018;103:2552-62. https://pubmed.ncbi.nlm.nih.gov/29897462/.

41. Benítez-Páez A, Gómez D, Pugar EM, López-Almela I, Moya-Pérez Á, Codoñer-Franch P, Sanz Y. Depletion of Blautia Species in the Microbiota of Obese Children Relates to Intestinal Inflammation and Metabolic Phenotype Worsening. mSystems. 2020;5:e00857-19. https://pubmed.ncbi.nlm.nih.gov/32209719/.

\section{Figures}




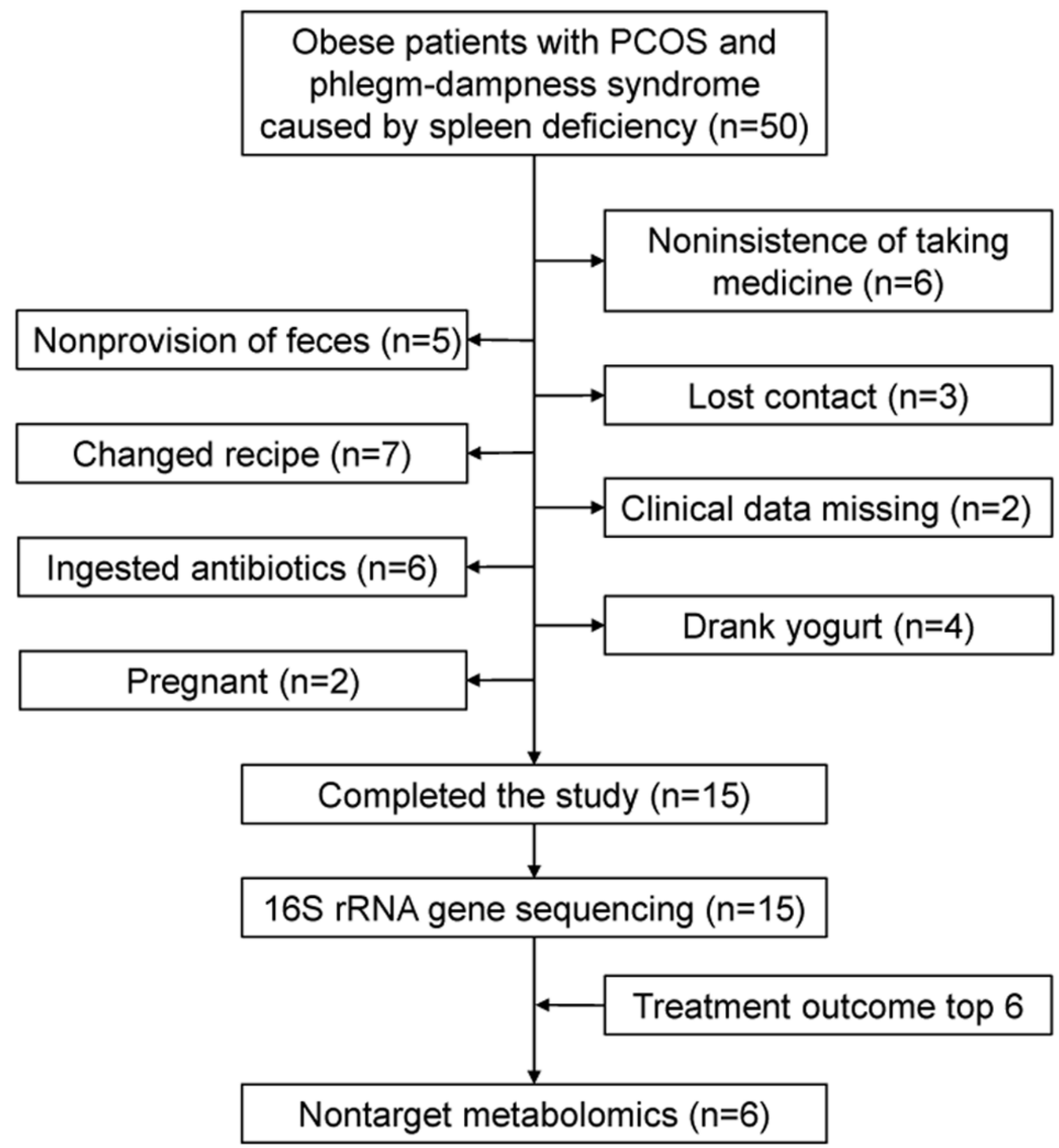

Figure 1

Flow chart of patients inclusion and exclusion. 


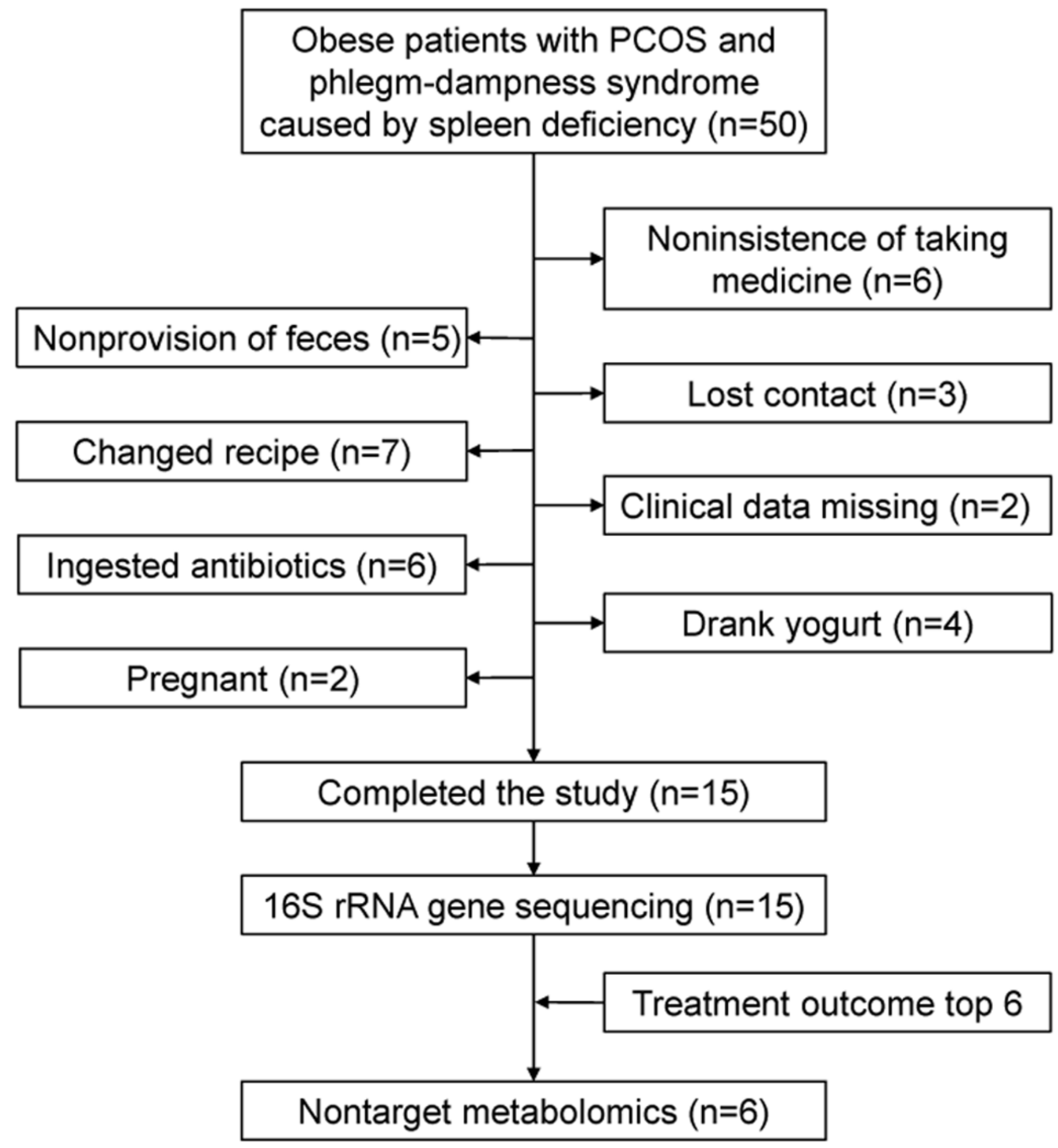

Figure 1

Flow chart of patients inclusion and exclusion. 

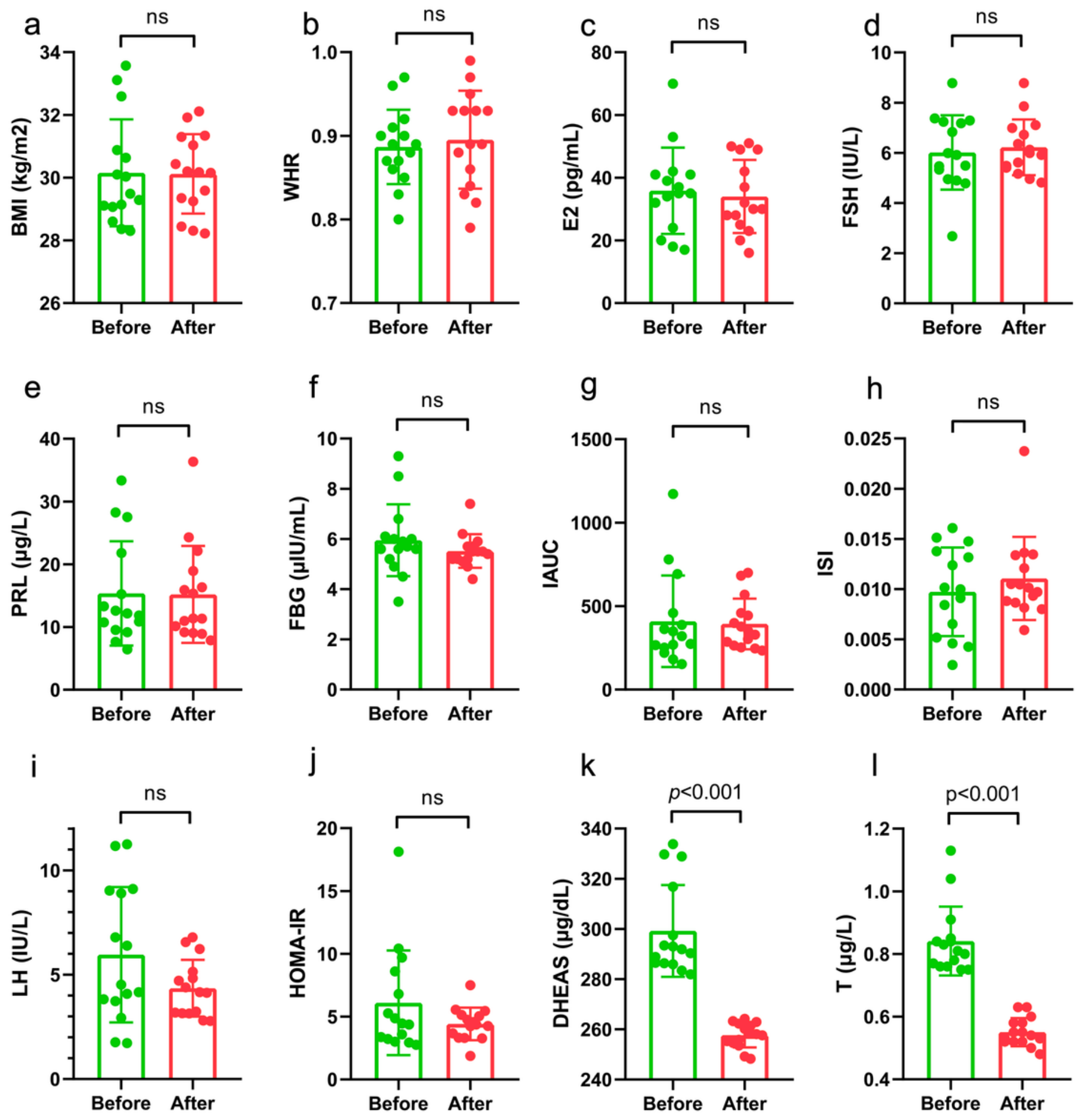

Figure 2

Clinical data of the patients before and after BZYQ treatment. (a) BMI, body mass index; (b) WHR, waistto-hip ratio; (c) E2, estradiol; (d) FSH, follicle-stimulating hormone; (e) PRL, prolactin; (f) FBG, fasting blood glucose; (g) IAUC, insulin area under the curve; (h) ISI, insulin sensitive index; (i) LH, luteinizing hormone; (j) HOMA-IR, homeostatic model assessment for insulin resistance; (k) DHEAS, dehydroepiandrosterone sulfate; (I) T, testosterone. ns, no significant. 

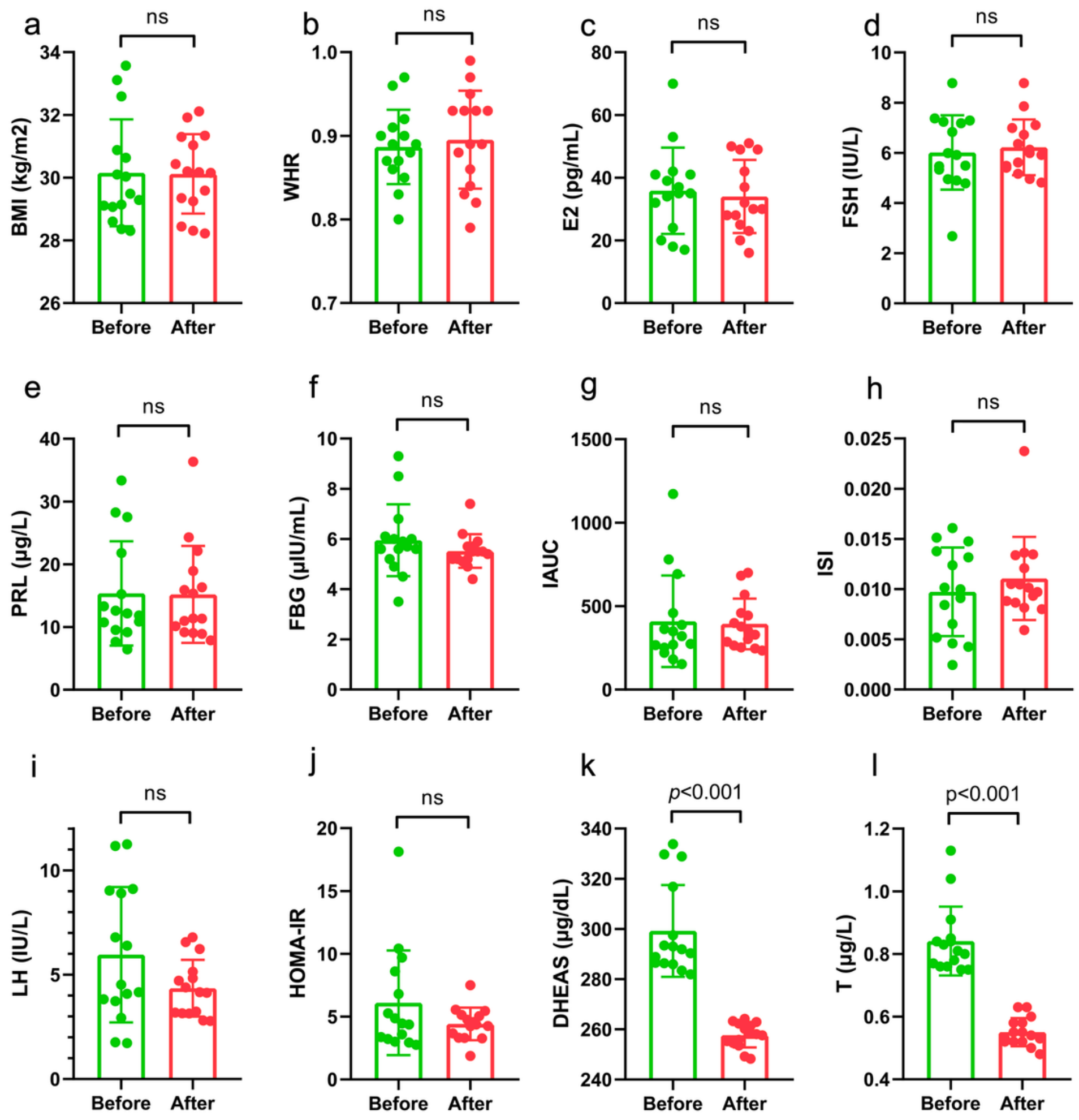

Figure 2

Clinical data of the patients before and after BZYQ treatment. (a) BMI, body mass index; (b) WHR, waistto-hip ratio; (c) E2, estradiol; (d) FSH, follicle-stimulating hormone; (e) PRL, prolactin; (f) FBG, fasting blood glucose; (g) IAUC, insulin area under the curve; (h) ISI, insulin sensitive index; (i) LH, luteinizing hormone; (j) HOMA-IR, homeostatic model assessment for insulin resistance; (k) DHEAS, dehydroepiandrosterone sulfate; (I) T, testosterone. ns, no significant. 
a
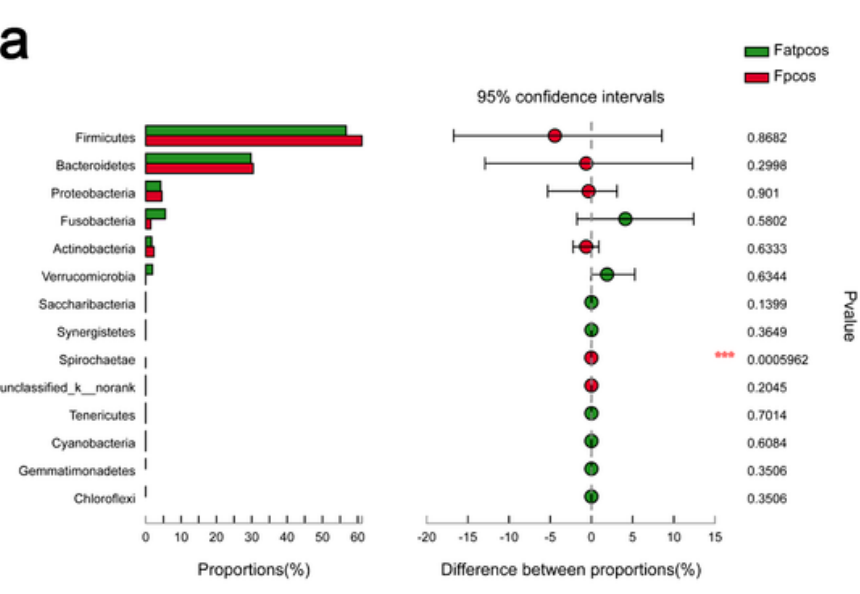

C

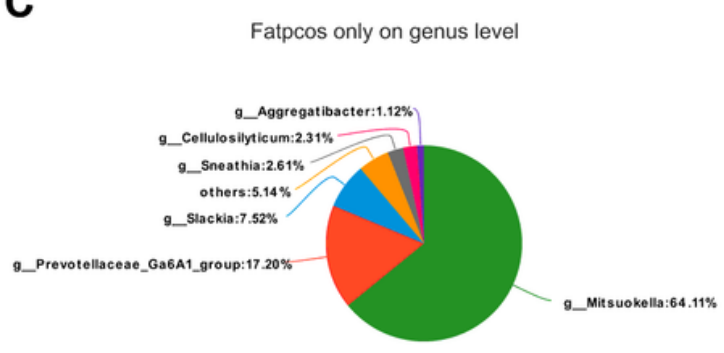

d

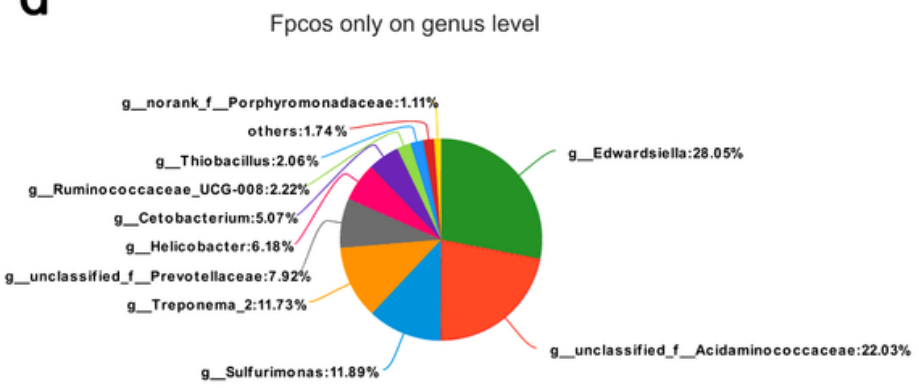

b
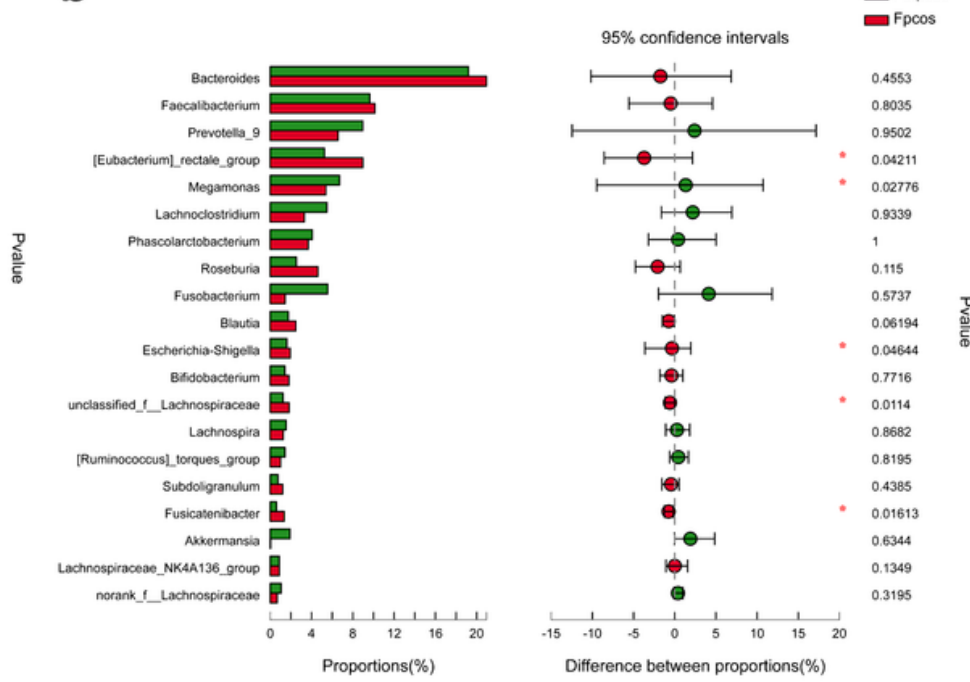

e

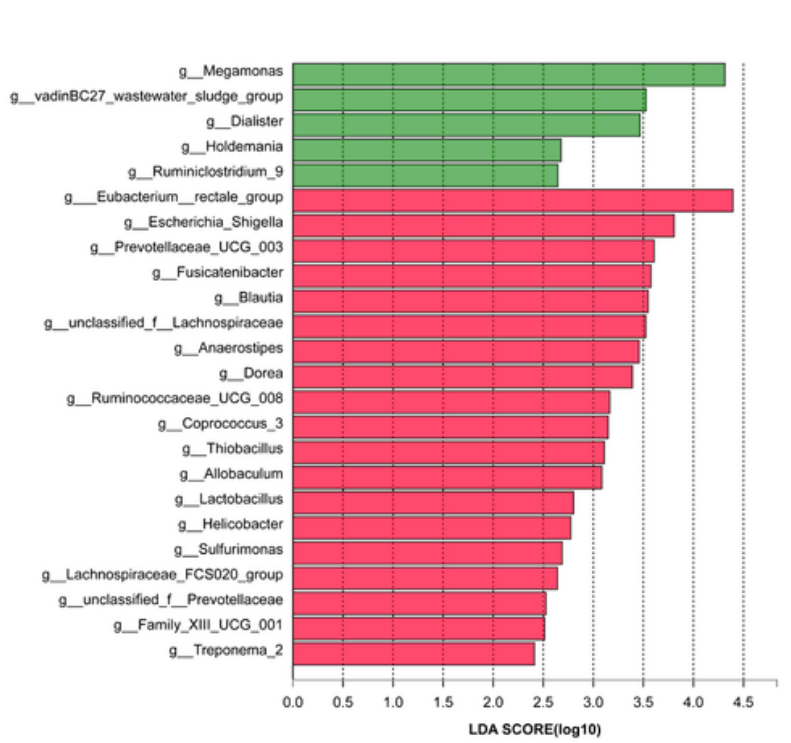

\section{Figure 3}

Structure of gut microbiota in patients before and after BZYQ treatment. Histogram of gut microbiota at phylum level (a) and genus level (top 20 in abundance) (b) before and after BZYQ treatment. On the left side is the mean relative abundance of species in the two groups; on the right side is the difference of species abundance before and after BZYQ treatment; boxes of different colors represent different groups. $\star, p<0.05$; $* \star \star, ~ p<0.01$; Fatpcos, before BZYQ treatment; Fpcos, after BZYQ treatment. Composition of abundance percentage of unique genera before (c) and after BZYQ treatment (d). (e) Linear discriminant analysis (LDA) discriminant column chart. The greater the LDA score, the greater the impact of representative species richness on the differences between groups. 
a

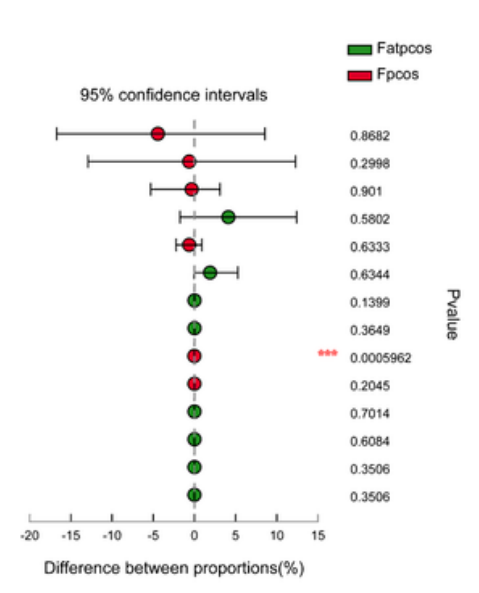

C

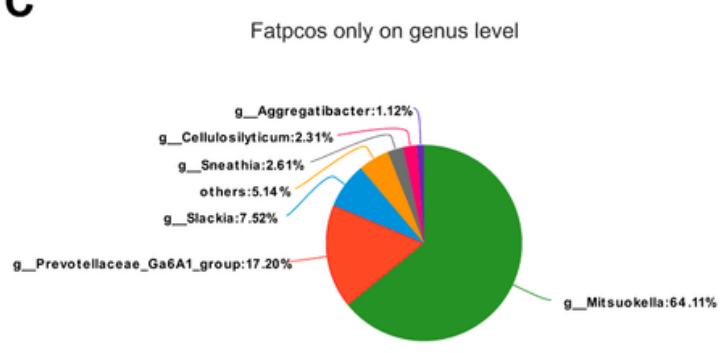

d

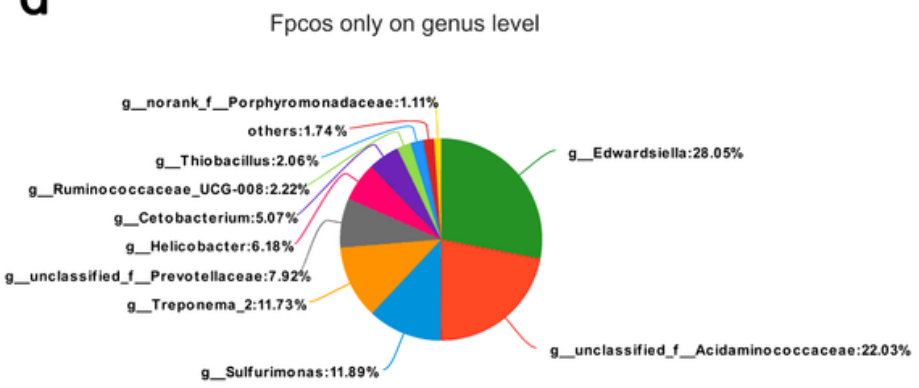

b
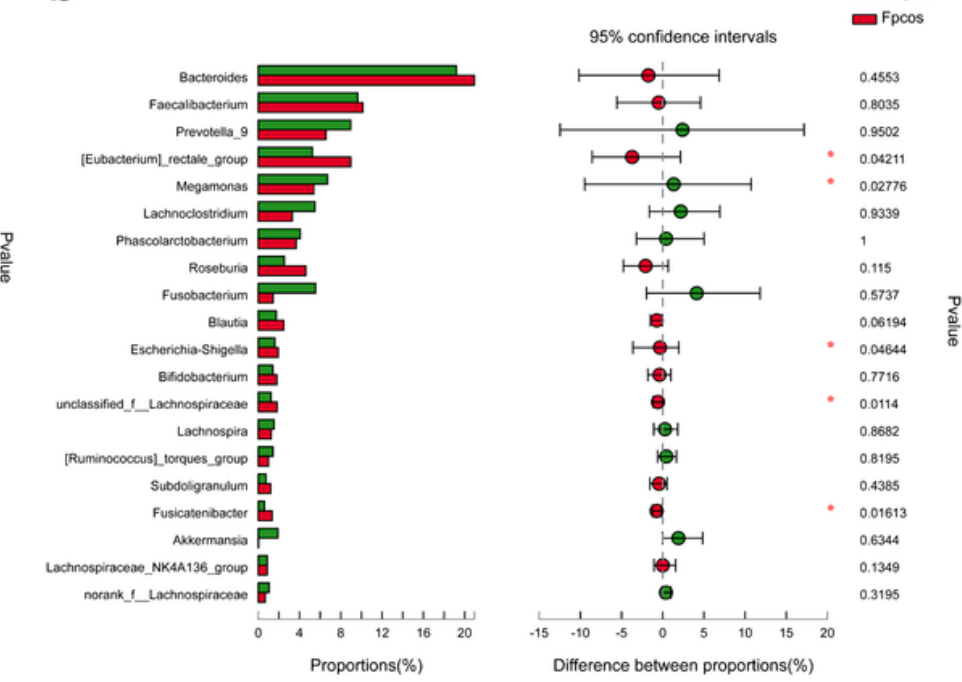

e

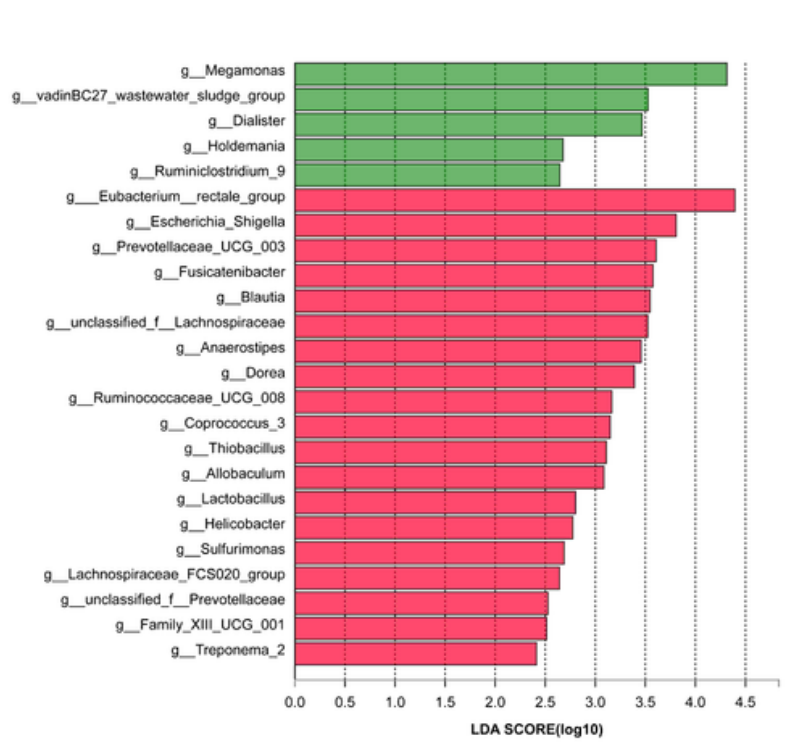

\section{Figure 3}

Structure of gut microbiota in patients before and after BZYQ treatment. Histogram of gut microbiota at phylum level (a) and genus level (top 20 in abundance) (b) before and after BZYQ treatment. On the left side is the mean relative abundance of species in the two groups; on the right side is the difference of species abundance before and after BZYQ treatment; boxes of different colors represent different groups. $\star, p<0.05$; $* \star \star, ~ p<0.01$; Fatpcos, before BZYQ treatment; Fpcos, after BZYQ treatment. Composition of abundance percentage of unique genera before (c) and after BZYQ treatment (d). (e) Linear discriminant analysis (LDA) discriminant column chart. The greater the LDA score, the greater the impact of representative species richness on the differences between groups. 
a

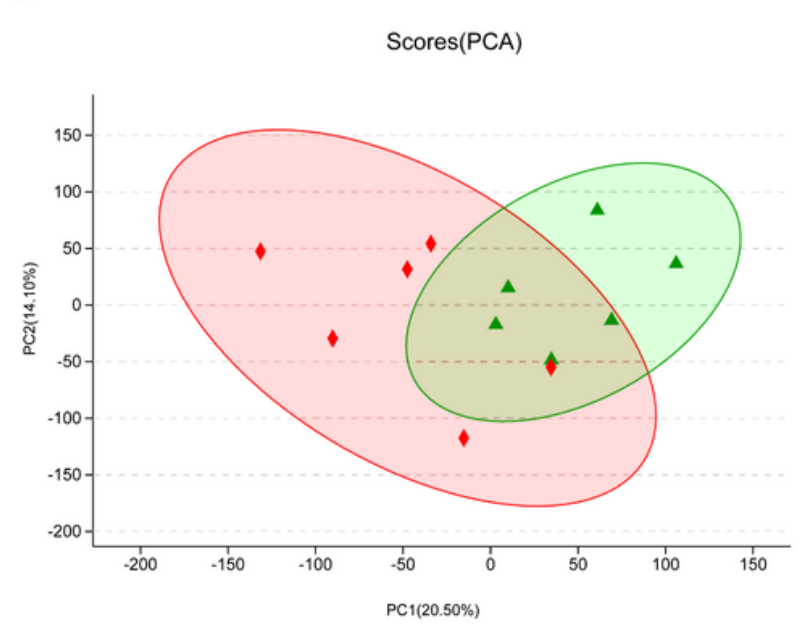

C

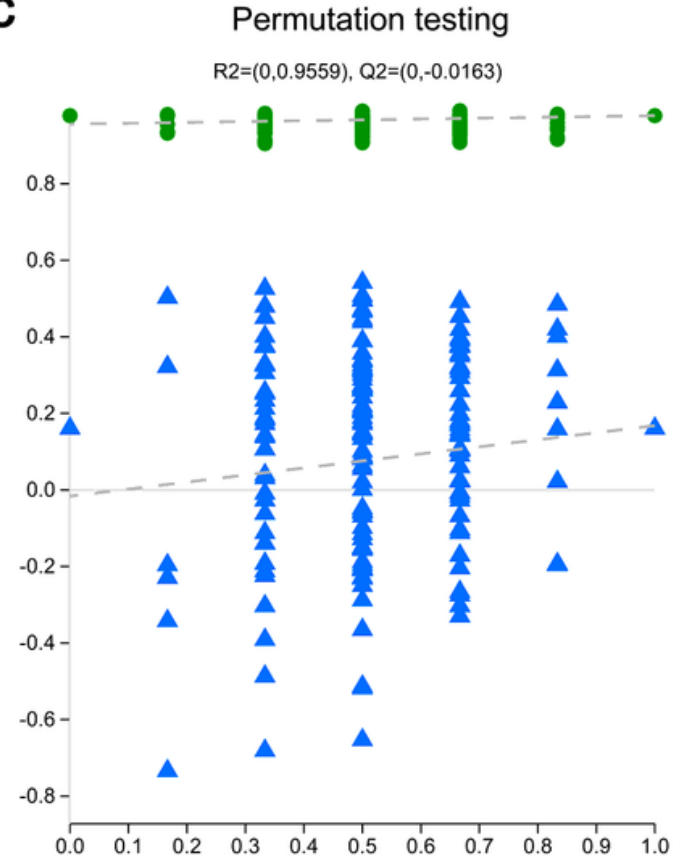

b
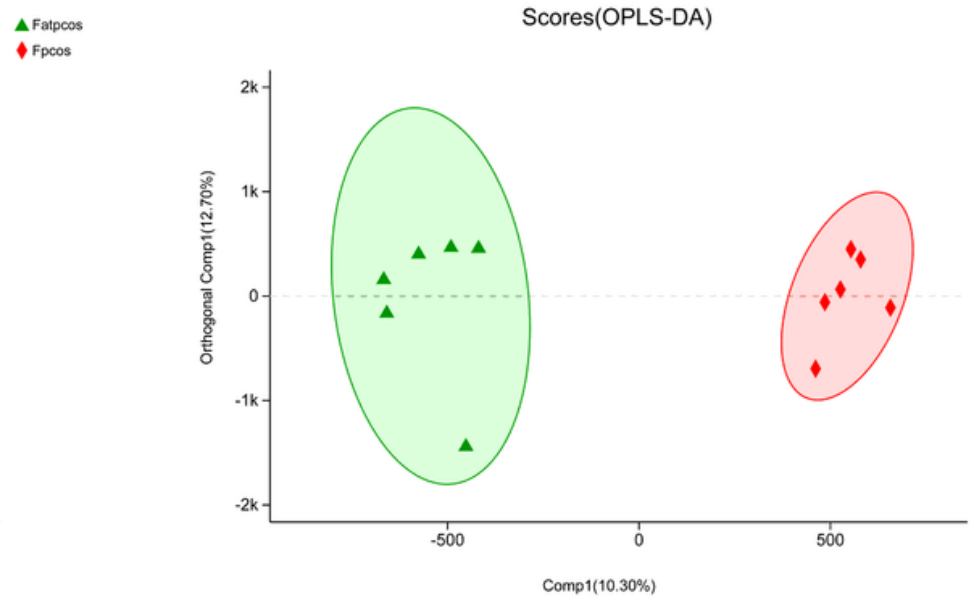

d

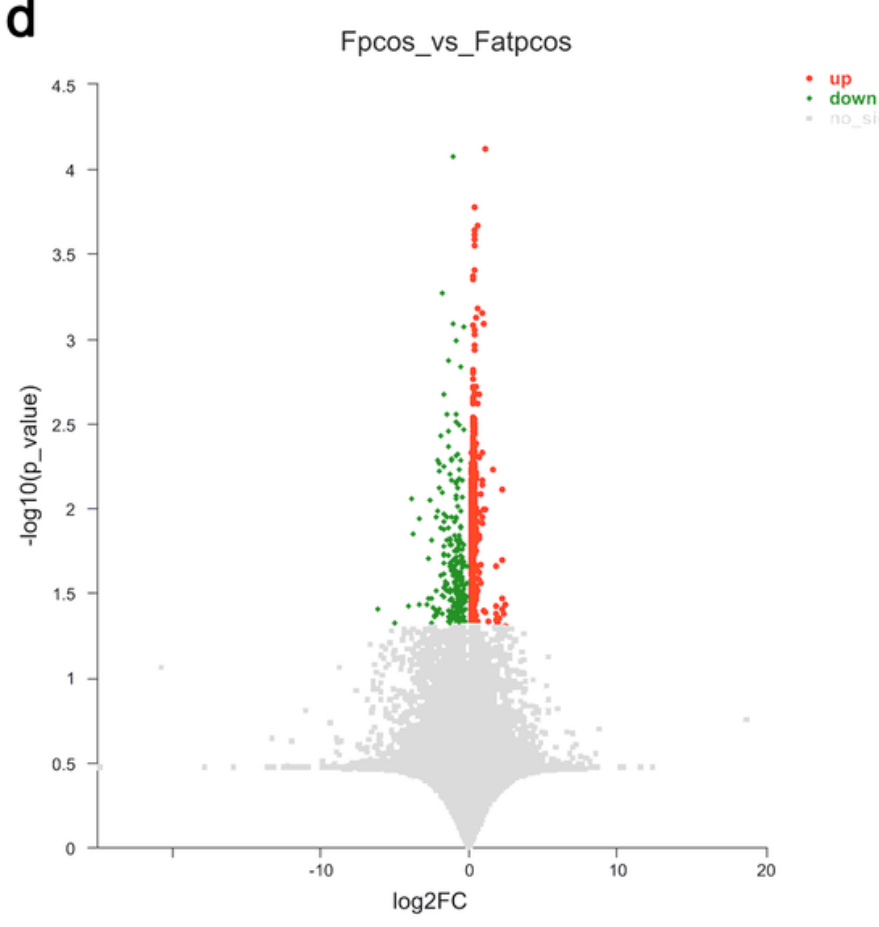

Figure 4

Fecal metabolites in patients before and after BZYQ treatment. PCA score chart (a) and OPLS-DA score chart (b) of fecal metabolites in obese PCOS patients before and after BZYQ treatment. Different color ellipses represent different groups. The distance between two points indicates the difference between two samples. (c) Model validation chart based on OPLS-DA of fecal metabolites in obese PCOS patients before and after BZYQ treatment. (d) Volcano map of fecal metabolites in obese PCOS patients before and after BZYQ treatment. Red indicates an increase in the level of metabolites, while green indicates a decrease in the level of metabolites. Fatpcos, before BZYQ treatment; Fpcos, after BZYQ treatment. 
a

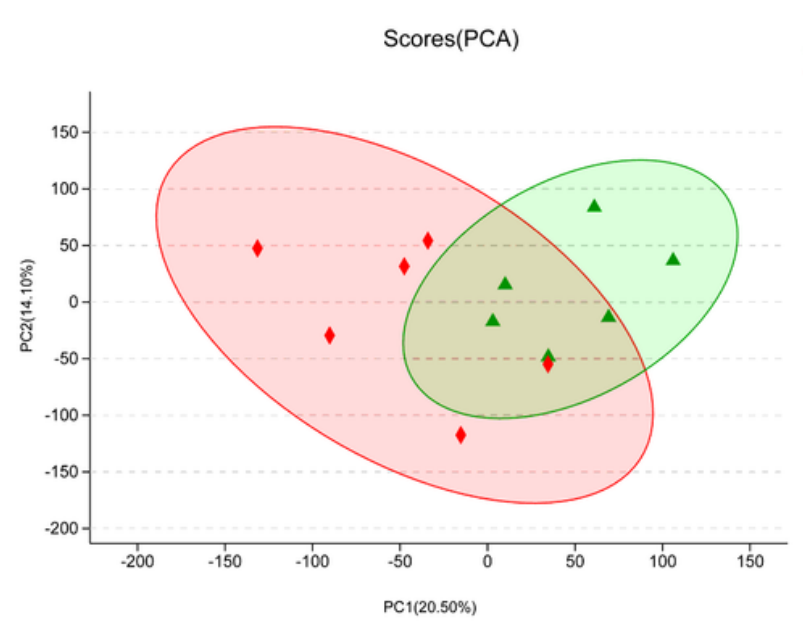

C

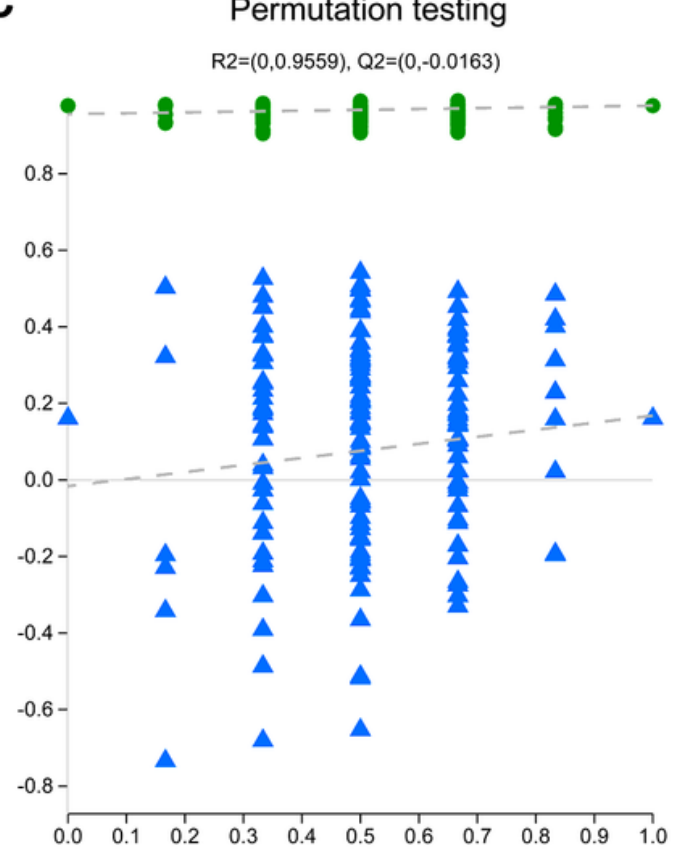

b
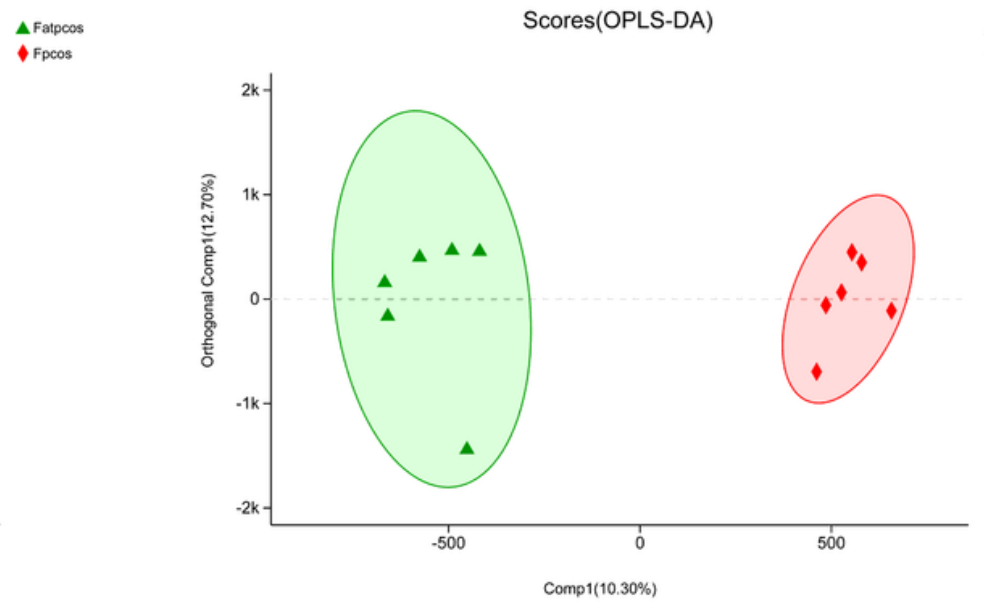

d

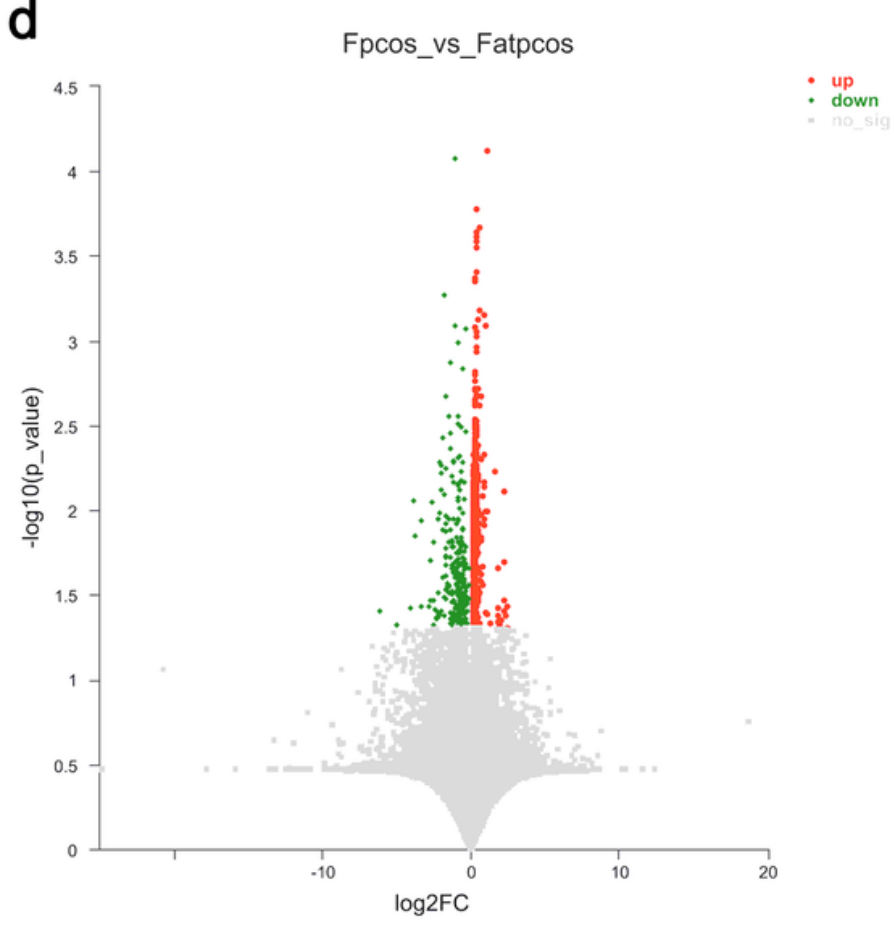

\section{Figure 4}

Fecal metabolites in patients before and after BZYQ treatment. PCA score chart (a) and OPLS-DA score chart (b) of fecal metabolites in obese PCOS patients before and after BZYQ treatment. Different color ellipses represent different groups. The distance between two points indicates the difference between two samples. (c) Model validation chart based on OPLS-DA of fecal metabolites in obese PCOS patients before and after BZYQ treatment. (d) Volcano map of fecal metabolites in obese PCOS patients before and after BZYQ treatment. Red indicates an increase in the level of metabolites, while green indicates a decrease in the level of metabolites. Fatpcos, before BZYQ treatment; Fpcos, after BZYQ treatment. 
a

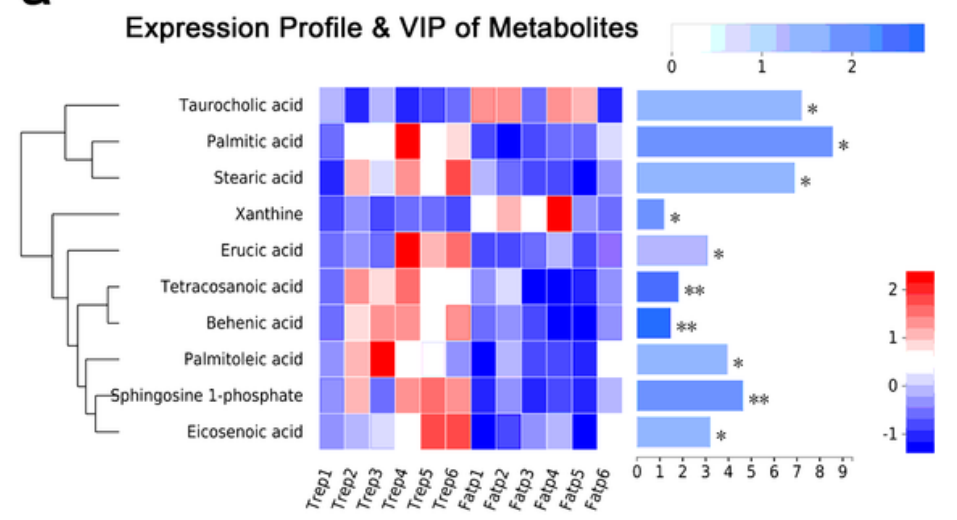

b

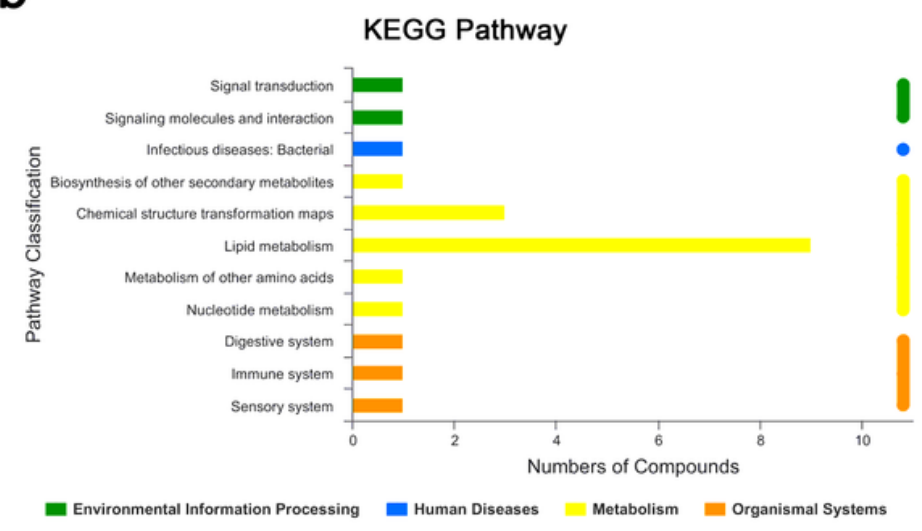

C

KEGG Enrichment Analysis

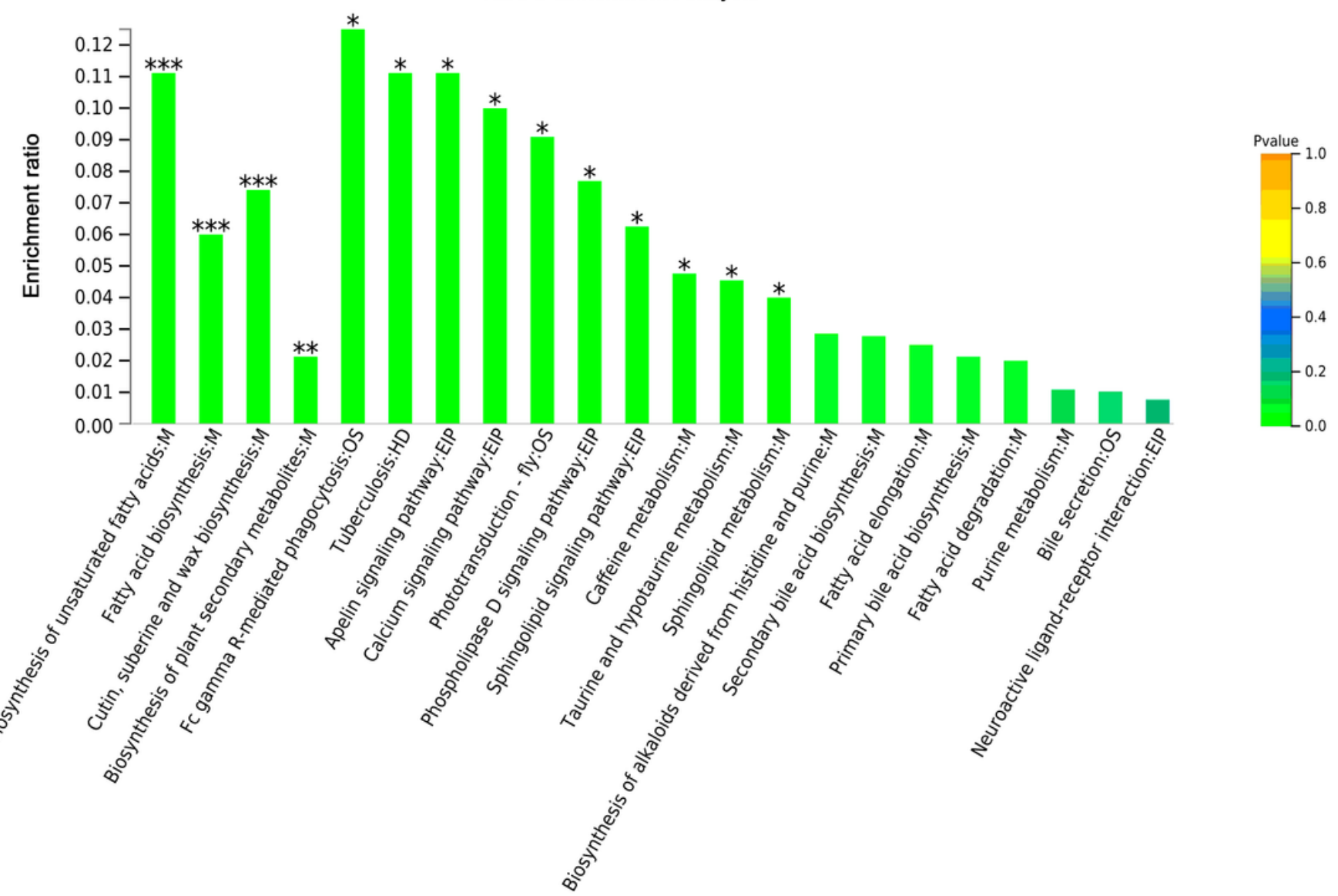

\section{Figure 5}

KEGG pathways related to differential fecal metabolites. (a) Heatmap of 10 differential metabolites involved in KEGG pathways. Each column represents a sample, and the bottom is the sample name; each row represents a metabolite, and the color represents the relative expression of the metabolite. On the right is the VIP bar graph of metabolites. The length of the bar represents the contribution value of the metabolites to the difference between the two groups. Trep 1-6, the samples after BZYQ treatment; Fatp 16 , the samples before BZYQ treatment; $*, p<0.05$; $* *, p<0.01$; $* \star *, p<0.001$. (b) Classification bar of KEGG pathway related to the differential metabolites. The ordinate is the name of KEGG pathway level 2 , and the abscissa is the number of differential metabolites annotated to the pathway. Different colors 
represent different KEGG pathway level 1. (c) Enrichment of KEGG pathways related to differential metabolites. The abscissa is the name of KEGG pathway level 3; the ordinate is the enrichment rate, indicating the ratio of the number of differential metabolites in this study enriched in the pathway to the number of metabolites annotated in the pathway. The larger the ratio, the greater the degree of enrichment. *, $\mathrm{p}<0.05 ; * *, \mathrm{p}<0.01 ; * * *, \mathrm{p}<0.001$.

a

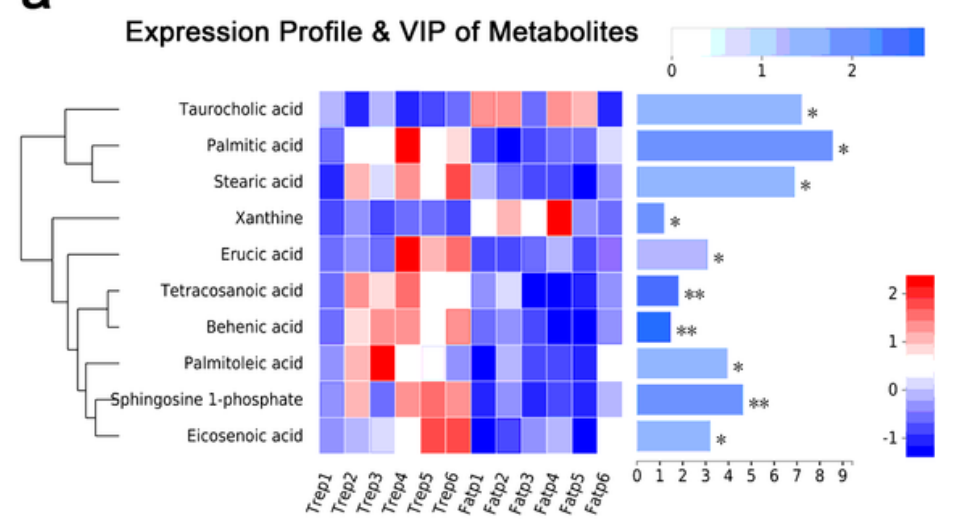

b

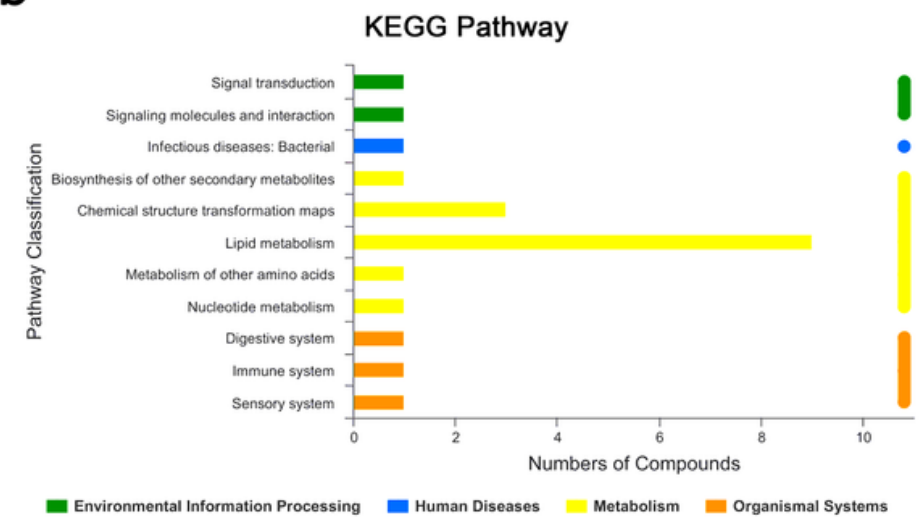

C

KEGG Enrichment Analysis
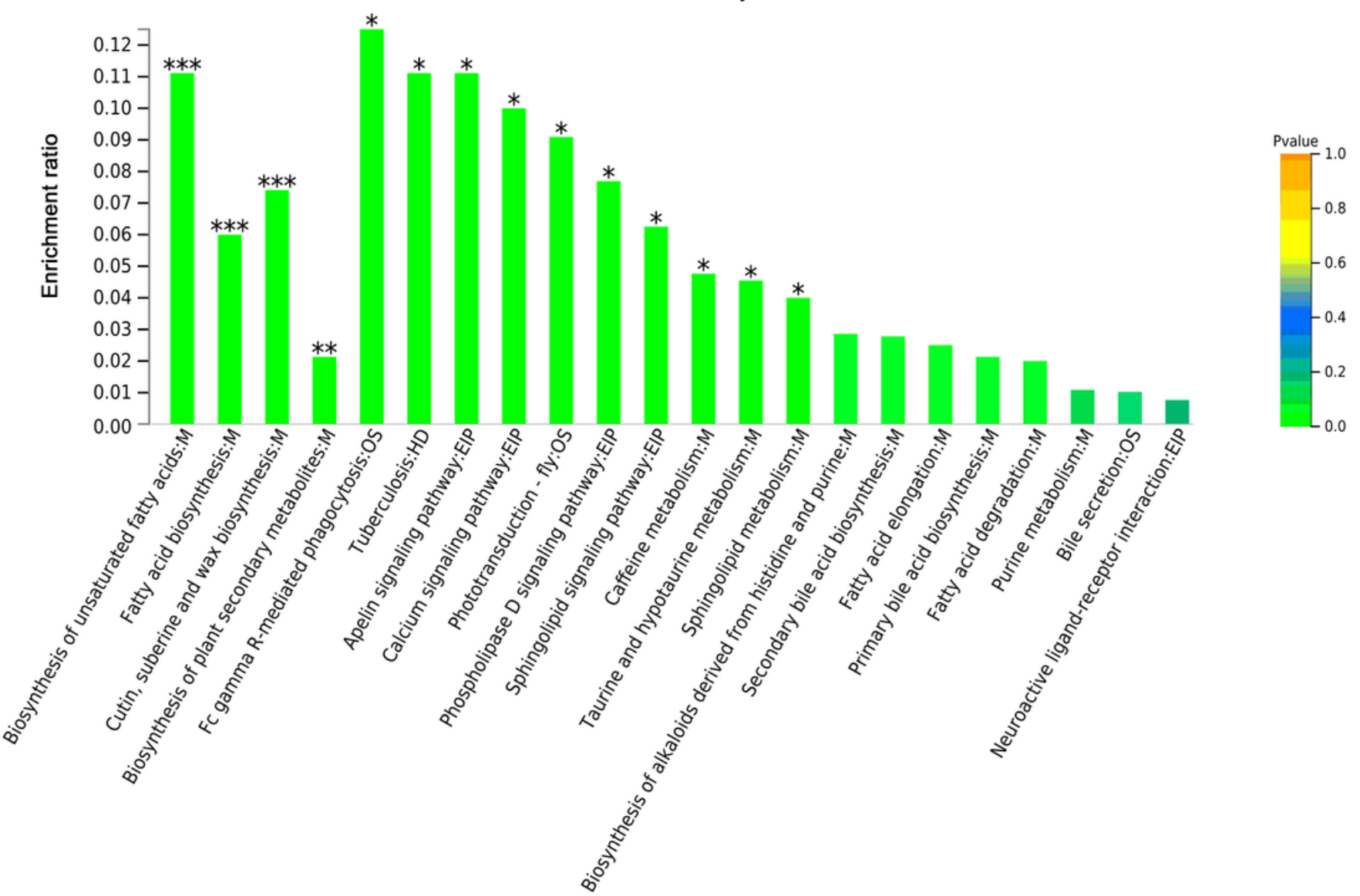

Figure 5

KEGG pathways related to differential fecal metabolites. (a) Heatmap of 10 differential metabolites involved in KEGG pathways. Each column represents a sample, and the bottom is the sample name; each row represents a metabolite, and the color represents the relative expression of the metabolite. On the 
right is the VIP bar graph of metabolites. The length of the bar represents the contribution value of the metabolites to the difference between the two groups. Trep 1-6, the samples after BZYQ treatment; Fatp 16 , the samples before BZYQ treatment; $*, p<0.05$; $* *, p<0.01$; $* \star *, p<0.001$. (b) Classification bar of KEGG pathway related to the differential metabolites. The ordinate is the name of KEGG pathway level 2, and the abscissa is the number of differential metabolites annotated to the pathway. Different colors represent different KEGG pathway level 1. (c) Enrichment of KEGG pathways related to differential metabolites. The abscissa is the name of KEGG pathway level 3; the ordinate is the enrichment rate, indicating the ratio of the number of differential metabolites in this study enriched in the pathway to the number of metabolites annotated in the pathway. The larger the ratio, the greater the degree of enrichment. *, $p<0.05 ; * *, p<0.01 ; * * *, p<0.001$. 


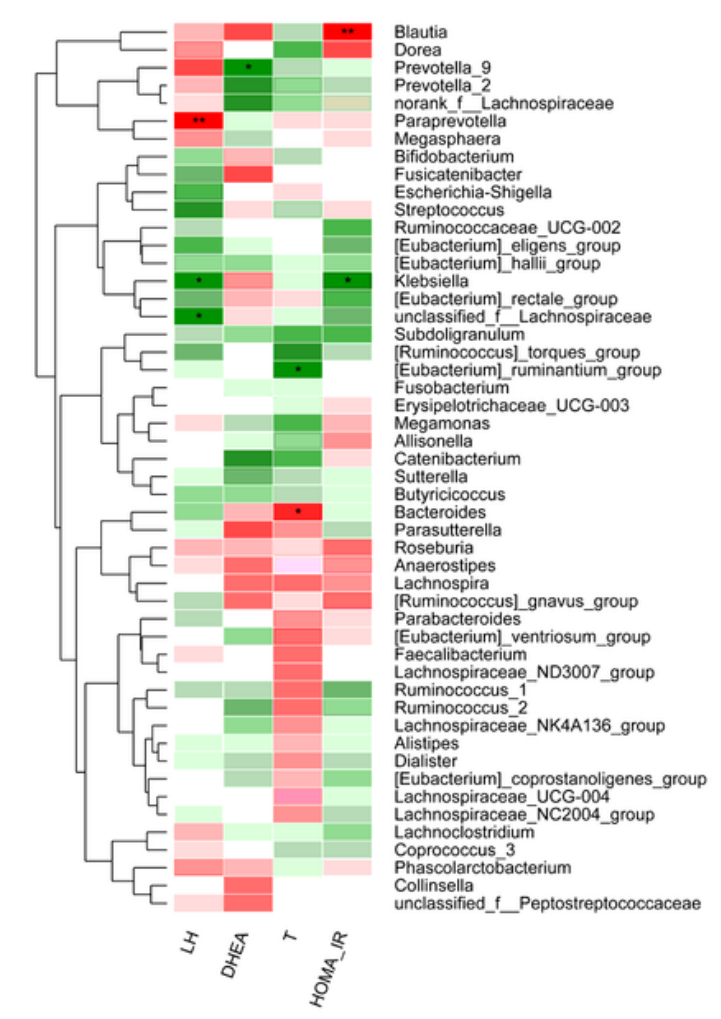

C

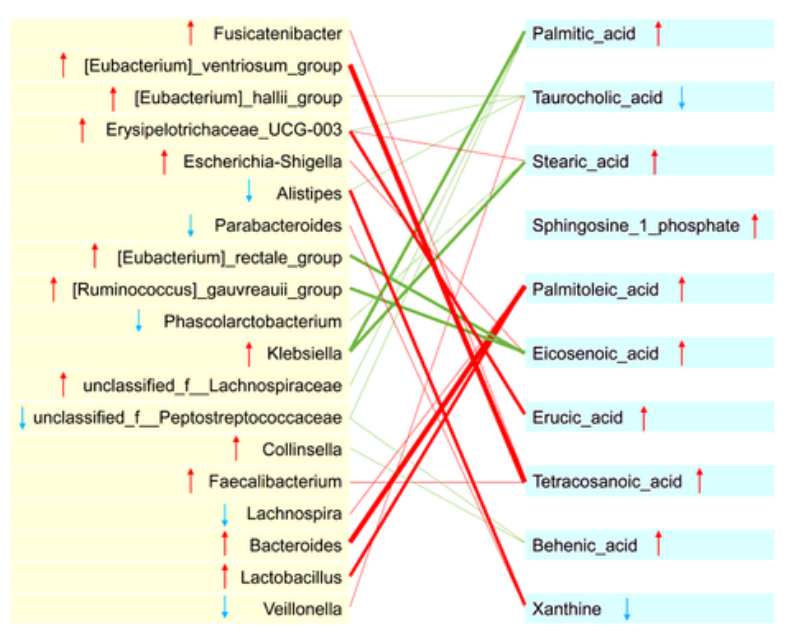

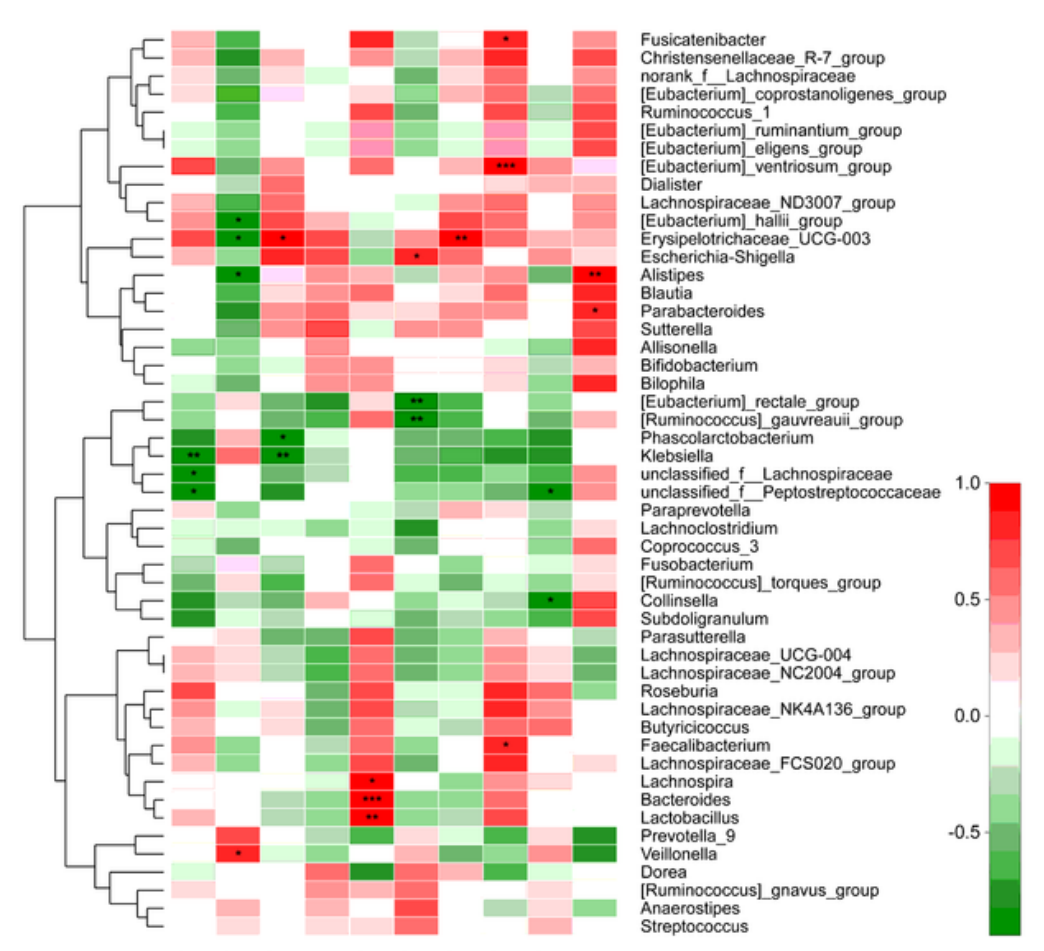

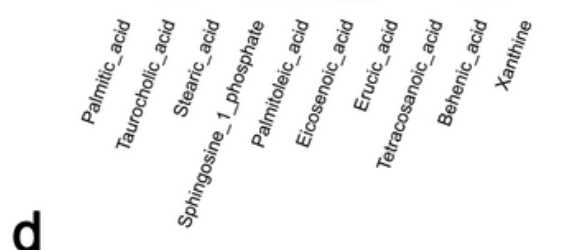

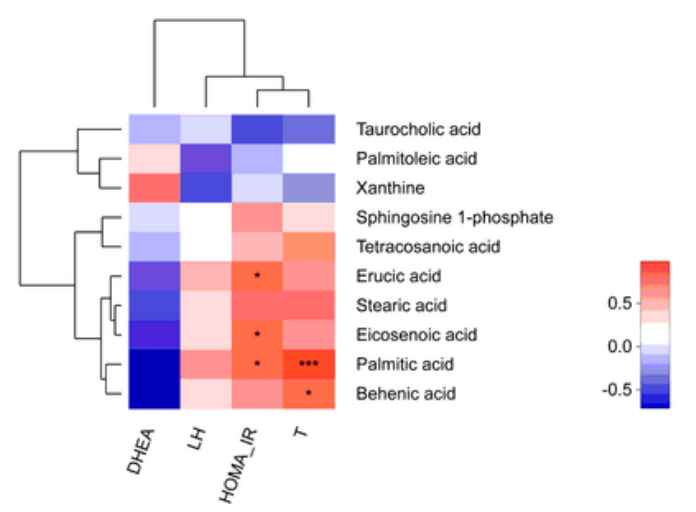

Figure 6

Correlation analysis among gut microbiota, fecal metabolites, and serum sex hormones. (a) Correlation heatmap of serum sex hormone, HOMA-IR and gut microbiota (top 50) after BZYQ treatment. (b) Correlation heatmap of fecal metabolites and gut microbiota (top 50) after BZYQ treatment. Red represents positive correlation, green represents negative correlation. (c) Correlation map of 10 differential metabolites involved in KEGG pathways and relative species at genus level. Red line represents positive correlation, and green line represents negative correlation; the thickness of the line 
represents the correlation degree, and the thicker the line is, the stronger the correlation is; the arrow up indicates the increase of the abundance, and down indicates the decrease of the abundance. (d) Correlation heatmap of serum sex hormone, HOMA-IR and 10 differential metabolites involved in KEGG pathways. ${ }^{*}, p<0.05, * *, p<0.01, * * *, p<0.001$

a

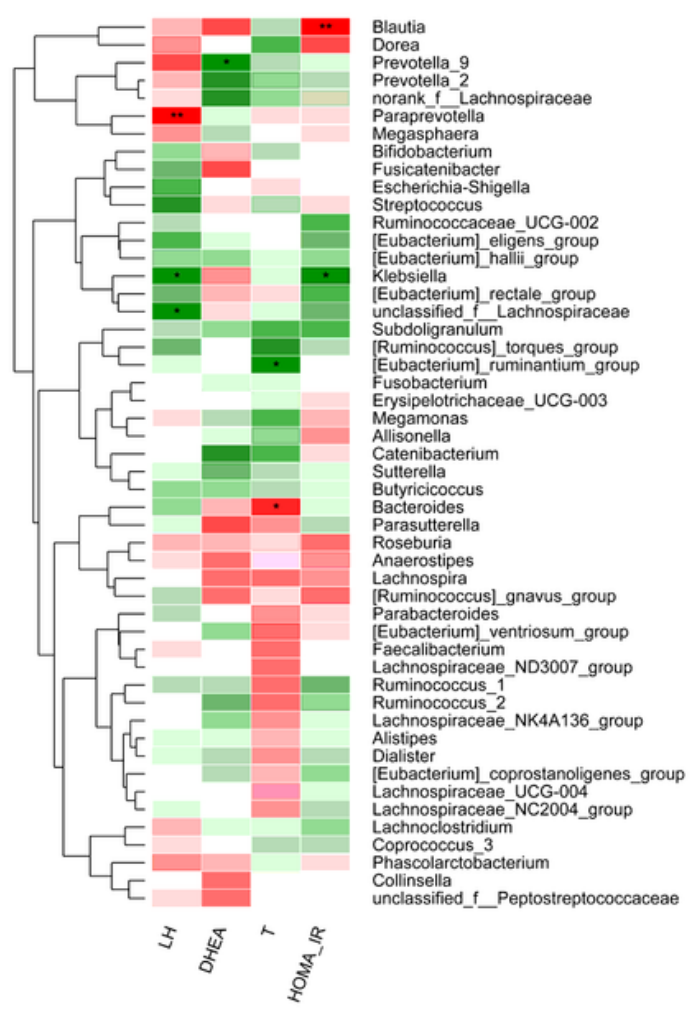

C

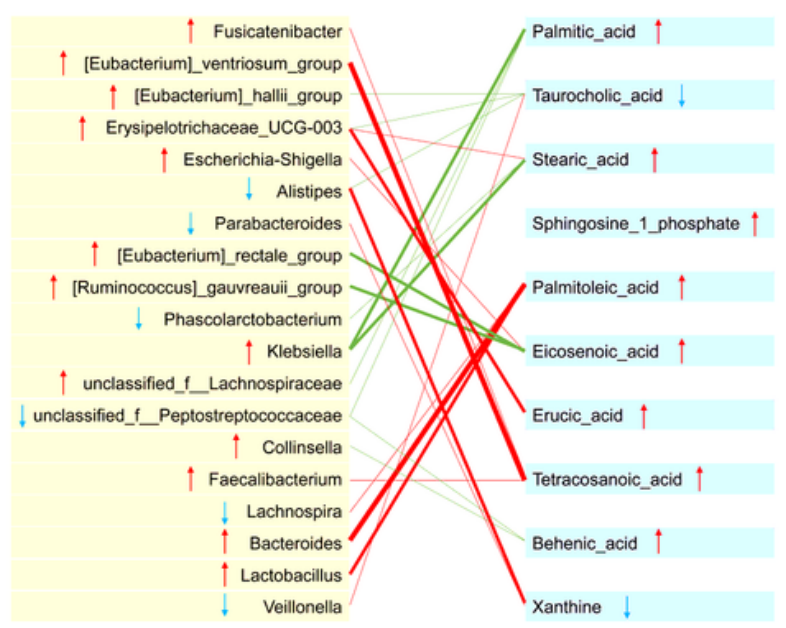

b
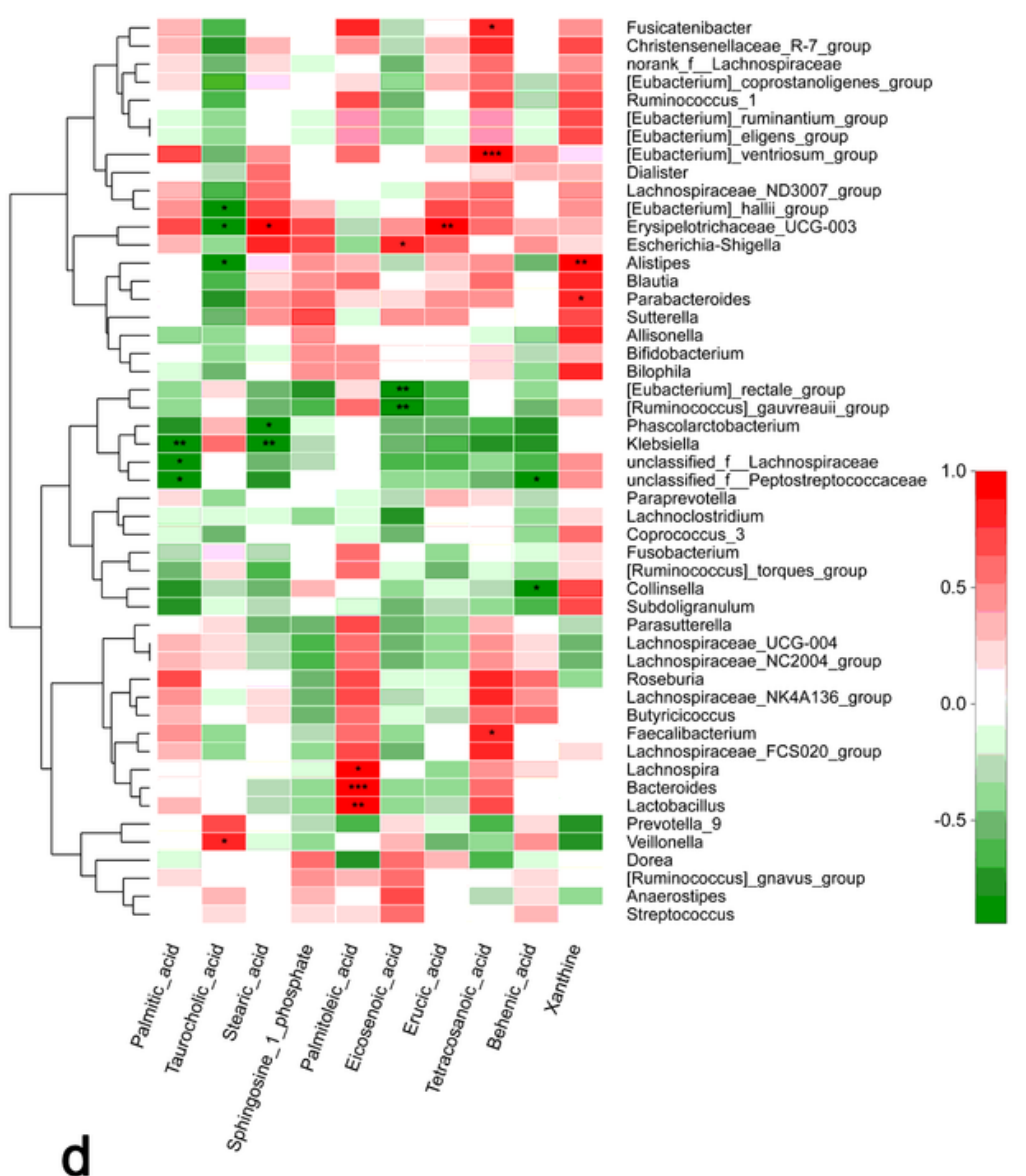
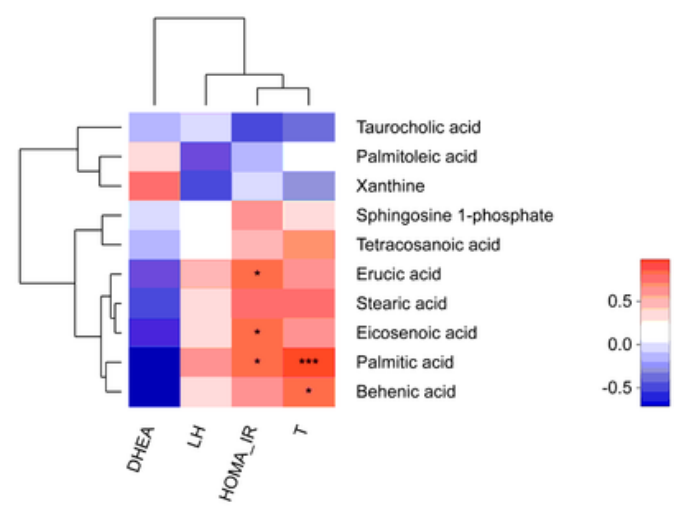

Figure 6

Correlation analysis among gut microbiota, fecal metabolites, and serum sex hormones. (a) Correlation heatmap of serum sex hormone, HOMA-IR and gut microbiota (top 50) after BZYQ treatment. (b) 
Correlation heatmap of fecal metabolites and gut microbiota (top 50) after BZYQ treatment. Red represents positive correlation, green represents negative correlation. (c) Correlation map of 10 differential metabolites involved in KEGG pathways and relative species at genus level. Red line represents positive correlation, and green line represents negative correlation; the thickness of the line represents the correlation degree, and the thicker the line is, the stronger the correlation is; the arrow up indicates the increase of the abundance, and down indicates the decrease of the abundance. (d) Correlation heatmap of serum sex hormone, HOMA-IR and 10 differential metabolites involved in KEGG pathways. ${ }^{*}, p<0.05,{ }^{*}, p<0.01, * \star *, p<0.001$.

\section{Supplementary Files}

This is a list of supplementary files associated with this preprint. Click to download.

- figureS.tif

- figures.tif 